Frontiers in Heat and Mass Transfer

\title{
HEAT TRANSFER BOUNDARY LAYER FLOW OF JEFFREY'S FLUID FROM A VERTICAL ISOTHERMAL CONE IN THE PRESENCE OF MICRO-POLAR
}

\author{
K. Madhavi ${ }^{a, b^{*}}$, V. Ramachandra Prasad ${ }^{\mathrm{a}}$, N. Nagendra ${ }^{\mathrm{a}}$, G.S.S. Raju ${ }^{\mathrm{b}}$ \\ ${ }^{a}$ Department of Mathematics, Madanapalle Institute of Technology and Science, Madanapalle-517325, India. \\ ${ }^{b}$ Department of Mathematics, JNTUA College of Engineering, Pulivendula-516390, Andhra Pradesh, India.
}

\begin{abstract}
In this article, the combined theoretical and computational study of the magneto hydrodynamic heat transfer in an electro-conductive polymer on the external surface of a vertical truncated cone under radial magnetic field is presented. Thermal and velocity (hydrodynamic) slip are considered at the vertical truncated cone surface via modified boundary conditions. The Williamson viscoelastic model is employed which is representative of certain industrial polymers. The governing partial differential equations (PDEs) are transformed into highly nonlinear, coupled, multi-degree non-similar partial differential equations consisting of the momentum and energy equations via appropriate non-similarity transformations. These transformed conservation equations are solved subject to appropriate boundary conditions with a second order accurate finite difference method of the implicit type. Validation of the numerical solutions is achieved via benchmarking with earlier published results. The influence of Williamson viscoelastic fluid parameter, magnetic body force parameter, Thermal and velocity (hydrodynamic) slip parameters, stream wise variable and Prandtl number on thermos-fluid characteristics are studied graphically. The model is relevant to the simulation of magnetic polymer materials processing.

Keywords: Natural convection, Boundary layer flow, Jeffrey fluid, Micropolar fluid, Keller-Box method, vertical isothermal cone.
\end{abstract}

\section{INTRODUCTION}

Natural convection possesses immense uses for researchers with its presence in both nature and engineering applications. Convection cells appear in nature, from the thermals that rise above the sun light-warmed land or water, which is fundamental to all weather systems. This can also be observed in the rising plume of hot-air from fire, ocean currents and sea-wind formation. Natural convection heat-transfer is widely employed in the areas of engineering such as: Cooling of commercial high-voltage electrical power transformers, Heating of houses by electrical baseboard heaters, Heat-loss from steam pipe lines in power plants and Heat-gain in refrigerant pipelines in air conditioning applications. It is also employed in the Cooling of reactor cores in nuclear power plants, although the coolant is often driven by pumps, resulting in more efficient heat transfer by forced convection, Refrigeration of electronic devices (chips, transistors) by finned heat sinks, although a fan is often used to segregate the natural convection with forced convection. In other studies, like fluid dynamics, nonNewtonian fluids play a very important role. In explaining the rheological properties of fluids, it may be said that the fluid thickness changes significantly with temperature.

Many authors have examined the vertical cone with natural convection heat transforms, such as Amanulla et al. (2017) studied the multiple slip effetcs on heat transfer in a jeffery fluid over an inclined vertical plate. Aurangzaib et al. (2016) employed the partial slip effect on mixed convection flow towards a permeable shrinking sheet with stagnation point. Satya Narayana and Venkateswarlu (2016) presented the MHD micropolar fluid past a vertical porous plate in the presence of thermal radiation. Siddiqi et al. (2017) investigated the MHD micropolar fluid flow over shrinking sheet with radiation. Hayat et al. (2014) studied the MHD stagnation point flow of Jeffrey's fluid flow in an isothermal cone due to convective boundary conditions. Rao et al. (2015) explained the non-isothermal wedge with flow of Jeffrey's fluid. Nasir et al. (2016) utilized the presence of the heat source with heat transfer of a couple stress fluids over an oscillator-stretching sheet. Sadia Siddiqi et al. (2017) reported the presence of thermal radiation with periodic MHD natural convection boundary layer problem obtained by the micro-polar fluid. Ram Reddy and Pradeepa (2015) presented the convective boundary condition are represented by a free convective flow along a permeable vertical plate of a micro-polar fluid. Ashmawy (2015) analyzed fully developed by the micro-polar with natural convection. Dulal Pala and Gopinath Mandal (2017) studied the micropolar with MHD effects of stretching sheet of nanofluids. Bourantas and Loukopoulos (2014) explained the MHD field in an inclined rectangular with transient, laminar and natural convection flow of a micropolar Nano fluid. Asia et al. (2016) explained the electrically showing micropolar fluid in a porous channel with contracting wall under the exploit of MHD. Hari et al. (2015) investigated the magnetic, material and viscosity parameters on natural convective flow along vertical walls in case of both asymmetric and symmetric cooling and heating of the walls. Rashad et al. (2014) have obtained a mixed convection in two - dimensional boundary layer flow of a micropolar fluid in a vertical plane with the effect of chemical reaction coupled with heat and mass transfer. Ahmad et al. (2012) investigated the laminar film flow of a micro-polar fluid with boundary layer of micropolar fluid. Nagendra et al. (2008) investigated Peristaltic motion of a power-law fluid in an asymmetric vertical channel. Isaac Lare Animasaun (2016) analyzed the horizontal linearly stretchable melting surface with mixed convection of micropolar fluid. Aparna et al. (2017) explained the flow of fluid with slow rotation in permeable sphere in micropolar fluid. Mishra et al. (2015) investigated the concentration of a double stratified micro-polar fluid in the presence of a magnetic field

\footnotetext{
${ }^{*}$ Corresponding author. Email: saimadhavi912@gmail.com
} 
with electrically conducting incompressible viscous fluid on a vertical plate. Naveed et al. (2016) studied radiation effect with curved stretching sheet of boundary layer flow of a viscous fluid. Srinivasa Charya and Mendu Upendar (2013) employed double stratified micropolar fluid with heat and mass transfer characteristics of the free convection on a vertical plane with a variable wall temperature and concentration. Ching - yang Cheng (2008) examined the micropolar fluids about the sphere with constant wall temperature and concentration by the natural convection of heat and mass transfer. Rawat et al. (2016) analyzed the non-darcy porous medium with nonlinear stretching sheet reacting micropolar fluid. Anwar Beg et al. (2011) studied the Isothermal sphere with micropolar regime with Soret/Dufour effects. Abid Hussanan et al. (2017) explained the micropolar fluid with nano particles in water and engine oil. Aurangzaib and Sharidan Shafie (2013) examined Unsteady MHD Stagnation-Point Flow with Heat and Mass Transfer in a Micropolar Fluid in the Presence of Thermophoresis and Suction/Injection. Vanita and Anand Kumar (2016) investigated natural convection vertical cone with effect of magnetic parameter. Ching-Yang Cheng (2008) examined the micro-polar fluids with natural convection heat transfer of a vertical truncated cone. Chang (2008) investigated the micro-polar fluid along a slender hollow cylinder in the wall conduction effect with heat transfer. Cheng (2008) examined a horizontal elliptical cylinder in a Newtonian with constant surface heat flux by the temperature dependent viscosity effects on the natural convection. Molla et al. (2009) studied the natural convection flow from a horizontal cylinder with uniform heat flux in the presence of heat generation. Baag et al. (2017) studied the micropolar fluid past a vertical flat plate with mixed convection stagnation point flow. Aurangzaib et al. (2016) analysis the Micropolar fluid flow and heat transfer over an exponentially permeable shrinking sheet. Amanulla et al. (2017) discussed thermal and momentum slip effects on hydromagnetic Convection flow of a Williamson fluid past a vertical truncated cone.

The objective of the present investigation intends to study heat transfer boundary layer flow of Jeffrey's fluid from a vertical isothermal cone in the presence of micro-polar fluid with suction effect is also considered. The sequential solutions for velocity, angular velocity and temperature fields are computed by using Keller's implicit finite difference box scheme. The effects of governing flow parameters on the flow, angular velocity, temperature, friction factor coefficient and local Nusselt number are discussed and presented here in through graphs and tables

\section{MATHEMATICAL MODEL}

Consider a 2-D study free convection boundary layer flow a of non-Newtonian Jeffrey fluid from a vertical isothermal cone in micropolar fluid regime. It is assumed that both the cone and Jeffrey fluid are maintained initially at the same temperature. Instantaneously it is raised to a temperature $T_{w}>T_{\infty}$, where the latter (ambient) temperature of the fluid is sustained constant. The basis of the coordinate system is placed at the vertex of the cone, where the $\mathrm{x}$ coordinate is measured along the slant surface of the cone from the origin and the $y$-coordinate is directed normal to the surface cone (See Fig.1). $T_{w}>T_{\infty}$ i.e. the ambient temperature of the fluid which remains unchanged.

$\boldsymbol{T}=-p \boldsymbol{I}+\boldsymbol{S}, \quad$ where $\boldsymbol{S}=\frac{\mu}{1+\lambda}\left(\dot{\gamma}+\lambda_{1} \ddot{\gamma}\right)$

Where a dot above quantity denotes the material time derivative, $P$ is pressure, $I$ is the identity tensor, $\mu$ is dynamic viscosity, $\lambda$ is the ratio of relaxation to retardation times, $\lambda_{1}$ is the retardation time and $\dot{\gamma}$ is the shear rate.

The Jeffrey's model deals a formulation for simulating elastic effects arising in non-Newtonian flows. The shear stress rate and gradient of shear stress rate are defined as follows

$$
\begin{aligned}
& \dot{\gamma}=\nabla V+(\nabla V)^{T} \\
& \ddot{\gamma}=\frac{d}{d t}(\dot{\gamma})=\frac{\partial}{\partial t}(\dot{\gamma})+(V . \nabla) \dot{\gamma}
\end{aligned}
$$

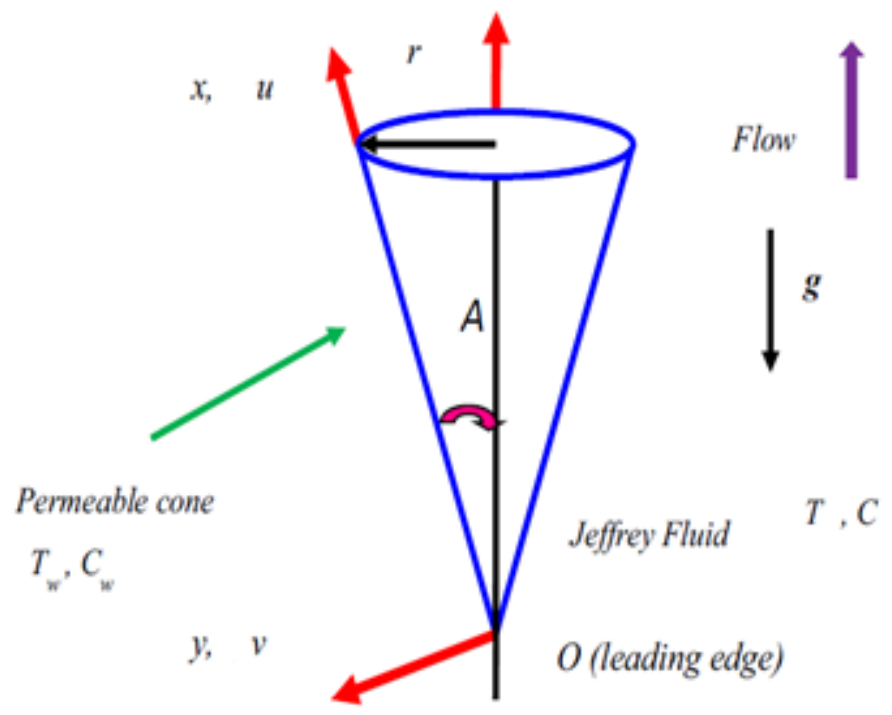

Fig. 1 Physical model and coordinate system

The Jeffery's model provides an elegant formulation for simulating retardation and relaxation effects arising in polymer flows. The outline of the appropriate terms in a flow model is considered next. The resulting boundary value problem is found to be well-posed and permits an excellent mechanism for the assessment of rheological characteristics of the flow behaviour.

We assume that the Boussinesq and boundary layer approximations, the governing equations for the conservation of mass, momentum, microrotation and energy can be written in two-dimensional Cartesian coordinates $(\mathrm{x}, \mathrm{y})$ which are relevant to the problem are as follows.

$\frac{\partial(r u)}{\partial x}+\frac{\partial(r v)}{\partial y}=0$

$\rho\left(u \frac{\partial u}{\partial x}+v \frac{\partial u}{\partial y}\right)=(\mu+k) \frac{\partial^{2} u}{\partial y^{2}}+\rho g \beta\left(T-T_{\infty}\right) \cos A+k \frac{\partial N}{\partial y}$

$+\frac{v}{1+\lambda}\left\{\frac{\partial^{2} u}{\partial y^{2}}+\lambda_{1}\left(u \frac{\partial^{3} u}{\partial x \partial y^{2}}-\frac{\partial u}{\partial x} \frac{\partial^{2} u}{\partial y^{2}}+\frac{\partial u}{\partial y} \frac{\partial^{2} u}{\partial x \partial y}+v \frac{\partial^{3} u}{\partial y^{3}}\right\}\right.$

$\rho_{j}\left(u \frac{\partial N}{\partial x}+v \frac{\partial N}{\partial y}\right)=\gamma^{*} \frac{\partial^{2} N}{\partial y^{2}}-k\left(2 N+\frac{\partial u}{\partial y}\right)$

$u \frac{\partial T}{\partial x}+v \frac{\partial T}{\partial y}=\alpha \frac{\partial^{2} T}{\partial y^{2}}$

Here $u$ and $v$ are the velocity components in the $x$-and $y$-directions respectively, $v$-the kinematic viscosity of the electrically conducting fluid.

The physically appropriate boundary conditions are as follows

$$
\begin{aligned}
& u=0 \quad v=-V_{w} \quad N=-\frac{1}{2} \frac{\partial u}{\partial y} \quad T=T_{w} \quad \text { at } \quad y=0 \\
& u \rightarrow 0 \quad v \rightarrow 0 \quad N \rightarrow 0 \quad T \rightarrow T_{\infty} \text { as } y \rightarrow \infty
\end{aligned}
$$

We introduce a stream function $\psi$ is defined by the CauchyRiemann equations

$r u=\frac{\partial \psi}{\partial y}$ and $r v=-\frac{\partial \psi}{\partial x}$ 
We introduce the non-dimensional variables

$$
\begin{aligned}
& \xi=\frac{\rho V_{w} x}{\mu}(G r)^{\frac{1}{4}} \eta=\frac{y}{x}(G r)^{\frac{1}{4}} g=\frac{\rho x^{2} N}{\mu} \\
& \theta(\xi, \eta)=\frac{T-T_{\infty}}{T_{w}-T_{\infty}} \psi=\frac{v r G r^{\frac{1}{4}}(f(\xi, \eta)+0.5 \xi)}{\rho}
\end{aligned}
$$

Substituting Eqn. (10) into the Eqns. (5)-(7), we obtain the following equations as

$$
\begin{aligned}
& \left\{\frac{4}{1+\lambda}+4(1+R)\right\} f^{\prime \prime \prime}+7 f f^{\prime \prime}+4 \theta+4 \xi f^{\prime \prime}+4 R g^{\prime} \\
& -2\left(f^{\prime}\right)^{2}-\frac{D e}{1+\lambda}\left\{2 f f^{\prime \prime \prime}-\left(f^{\prime \prime}\right)^{2}+7 f f^{i v}+4 \xi f^{i v}\right\} \\
& =\xi\left\{\begin{array}{l}
f^{\prime} \frac{\partial f^{\prime}}{\partial \xi}-f^{\prime \prime} \frac{\partial f}{\partial \xi} \\
-\frac{D e}{1+\lambda}\left(f^{\prime} \frac{\partial f^{\prime \prime \prime}}{\partial \xi}-f^{\prime \prime \prime} \frac{\partial f^{\prime}}{\partial \xi}+f^{\prime \prime} \frac{\partial f^{\prime \prime}}{\partial \xi}-f^{i v} \frac{\partial f}{\partial \xi}\right)
\end{array}\right\}
\end{aligned}
$$

$4\left(1+\frac{R}{2}\right) g^{\prime \prime}-4 R B \xi^{2}\left(2 g+f^{\prime \prime}\right)$

$+7 f g^{\prime}-f^{\prime} g+4 \xi g^{\prime}=\xi\left[f^{\prime} \frac{\partial g}{\partial \xi}-g^{\prime} \frac{\partial f}{\partial \xi}\right]$

$\frac{4}{\operatorname{Pr}} \theta^{\prime \prime}+7 f \theta^{\prime}+4 \xi \theta^{\prime}=\xi\left\{f^{\prime} \frac{\partial \theta}{\partial \xi}-\theta^{\prime} \frac{\partial f}{\partial \xi}\right\}$

Then the corresponding dimensionless boundary conditions becomes

At $\eta=0, f=0=f^{\prime}, g=\frac{1}{2} \frac{\partial^{2} f}{\partial y^{2}}, \theta=1$

As $\eta \rightarrow \infty, f^{\prime} \rightarrow 0, f^{\prime \prime} \rightarrow 0, g \rightarrow 0, \theta \rightarrow 0$

The skin-friction coefficient and the local Nusselt number (heat transfer rate) can be defined using the transformations described above with the following expressions:

$\frac{1}{2} G r_{x}^{-1 / 4} C_{f}=(2+R / 2) \xi f^{\prime \prime}(\xi, 0)+R g$

$G r_{x}^{-1 / 4} N u=-\theta^{\prime}(\xi, 0)$

\section{NUMERICAL SOLUTION WITH KELLER BOX IMPLICT METHOD}

The efficient Keller-box implicit difference method is implemented to solve the non-linear boundary layer eqns. (11) - (13) subject to the boundary conditions (14). This method originally developed for low speed aerodynamic boundary layers by Keller has been employed in a diverse range of nonlinear MHD and coupled heat transfer problems. It includes pressure work effect on natural convection flow from a vertical circular cone with suction and nonuniform surface temperature. Very few of these papers, It has been used recently in

polymeric flow dynamics by Amanulla et al. (2017) for viscoelastic models. However, have provided guidance for researchers as to customization of the Keller-Box scheme to heat transfer problems.

The fundamental phases intrinsic to the Keller-Box scheme are

a. Reduction of the Nth order partial differential equation system of $\mathrm{N}$ first order equations

b. Finite Difference Discretization

c. Quasilinearization of Non-Linear Keller algebraic Equations

d. Block-tridiagonal elimination of Linear Keller algebraic Equations
Phase a: Reduction of the nth order partial differential equation system to $\mathbf{N}$ first order equations

New variables are introduced to Eqns. (11)-(13) subject to the boundary conditions (14) are first written as a system of first order equations. For this purpose, we reset Eqns. (11) - (13) as a set of simultaneous equations by introducing the new variables

$$
\begin{aligned}
& f^{\prime}=u, u^{\prime}=v, v^{\prime}=q, q^{\prime}=f^{\prime v}, g^{\prime}=p, s^{\prime}=t \\
& \left\{\frac{4}{1+\lambda}+4(1+R)\right\} v^{\prime}+7 f v+4 R P-2 u^{2}+4 s+4 \xi v \\
& -A\left\{2 u q-v^{2}+7 f q^{\prime}+4 \xi q^{\prime}\right\}=\xi\left[\left(u \frac{\partial u}{\partial \xi}-v \frac{\partial f}{\partial \xi}\right)\right] \\
& -\xi\left[A\left(u \frac{\partial q}{\partial \xi}-q \frac{\partial u}{\partial \xi}+v \frac{\partial v}{\partial \xi}-q^{\prime} \frac{\partial f}{\partial \xi}\right)\right] \\
& 4\left(1+\frac{R}{2}\right) p^{\prime}-4 R B \xi^{2}(2 m+v)+7 f p-u m+4 \xi p \\
& =\left(u \frac{\partial m}{\partial \xi}-p \frac{\partial f}{\partial \xi}\right) \\
& \frac{4}{\operatorname{Pr}} t^{\prime}+7 f t+4 \xi t=\xi\left\{u \frac{\partial s}{\partial \xi}-t \frac{\partial f}{\partial \xi}\right\}
\end{aligned}
$$

Where primes denote differentiation $\eta$. In terms of the dependent variables, the boundary conditions become:

$$
\begin{aligned}
& f=0, u=0, s=1, g=-\frac{1}{2} v \text { at } \eta=0 \\
& u \rightarrow 0, v \rightarrow 0, s \rightarrow 0, g \rightarrow 0 \text { as } \eta \rightarrow \infty
\end{aligned}
$$

Phase b: Finite difference discretization of reduced boundary layer equations

A two-dimensional computational grid (mesh) is imposed on the $\xi-\eta$ plane as sketched in Fig.2. The stepping process is defined by:

$$
\begin{aligned}
& \eta_{0}=0, \eta_{j}=\eta_{j-1}+h_{j}, j=1,2, \ldots, J, \eta_{J} \equiv \eta_{\infty} \\
& \xi^{0}=0, \xi^{n}=\xi^{n-1}+k_{n}, n=1,2, \ldots, N
\end{aligned}
$$

Where $k_{n}$ and $h_{j}$ denote the step distances in the $\xi$ (stream wise) and $\eta$ (span wise) directions respectively

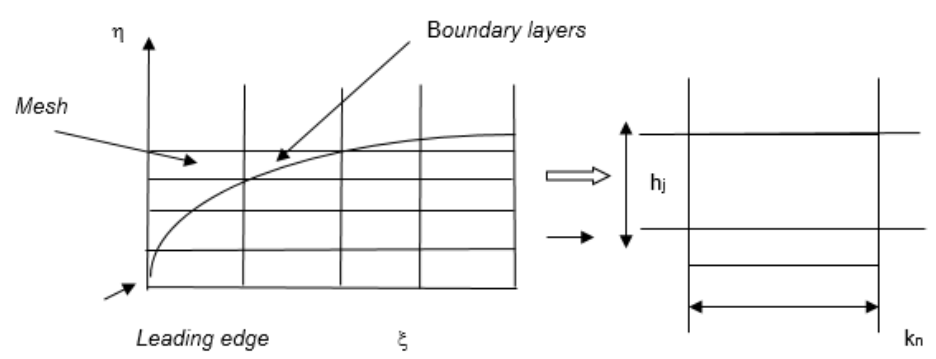

Fig. 2 Keller Box element and boundary layer mesh

If $g_{j}^{n}$ denotes the value of any variable at $\left(\eta_{j}, \xi^{n}\right)$, then the variables and derivatives of Equations. (17) - (20) at $\left(\eta_{j-1 / 2}, \xi^{n-1 / 2}\right)$ are replaced by

$g_{j-\frac{1}{2}}^{n-\frac{1}{2}}=\frac{1}{4}\left(g_{j}^{n}+g_{j-1}^{n}+g_{j}^{n-1}+g_{j-1}^{n-1}\right)$ 


$$
\begin{aligned}
& \left(\frac{\partial g}{\partial \eta}\right)_{j-\frac{1}{2}}^{n-\frac{1}{2}}=\frac{1}{2 h_{j}}\left(g_{j}^{n}-g_{j-1}^{n}+g_{j}^{n-1}-g_{j-1}^{n-1}\right) \\
& \left(\frac{\partial g}{\partial \xi}\right)_{j-\frac{1}{2}}^{n-\frac{1}{2}}=\frac{1}{2 k^{n}}\left(g_{j}^{n}-g_{j-1}^{n}+g_{j}^{n-1}-g_{j-1}^{n-1}\right)
\end{aligned}
$$

We now state the finite-difference approximation of Eqns. (17)-(20) for the mid-point $\left(\eta_{j-1 / 2}, \xi^{n}\right)$, below

$$
\begin{aligned}
& h_{j}^{-1}\left(f_{j}^{n}-f_{j-1}^{n}\right)=u_{j-\frac{1}{2}}^{n} \\
& h_{j}^{-1}\left(u_{j}^{n}-u_{j-1}^{n}\right)=v_{j-\frac{1}{2}}^{n} \\
& h_{j}^{-1}\left(v_{j}^{n}-v_{j-1}^{n}\right)=q_{j-\frac{1}{2}}^{n} \\
& h_{j}^{-1}\left(g_{j}^{n}-g_{j-1}^{n}\right)=p_{j-\frac{1}{2}}^{n} \\
& h_{j}^{-1}\left(\theta_{j}^{n}-\theta_{j-1}^{n}\right)=t_{j-\frac{1}{2}}^{n} \\
& \left\{\frac{4}{1+\lambda}+4(1+R)\right\}\left(\frac{v_{j}-v_{j-1}}{h_{j}}\right)-4 A \xi\left(\frac{q_{j}-q_{j-1}}{h_{j}}\right) \\
& +\frac{(\alpha+7)}{4}\left(f_{j}+f_{j-1}\right)\left(v_{j}+v_{j-1}\right)+2\left(s_{j}+s_{j-1}\right) \\
& +2 R\left(p_{j}+p_{j-1}\right)-\frac{(\alpha+2)}{4}\left(u_{j}+u_{j-1}\right)^{2}+2 \xi\left(v_{j}+v_{j-1}\right) \\
& +\frac{A(1+\alpha)}{4}\left(v_{j}+v_{j-1}\right)^{2}-\frac{A}{2}\left(u_{j}+u_{j-1}\right)\left(q_{j}-q_{j-1}\right) \\
& -\frac{A(7+\alpha)}{2}\left(f_{j}+f_{j-1}\right)\left(\frac{q_{j}-q_{j-1}}{h_{j}}\right)+\frac{\alpha}{2}\left(f_{j}+f_{j-1}\right) v^{n-1} \\
& -\frac{\alpha}{2}\left(v_{j}+v_{j-1}\right) f^{n-1}-A \alpha\left(u_{j}+u_{j-1}\right) q^{n-1}+A \alpha\left(q_{j}+q_{j-1}\right) u^{n-1} \\
& +A \alpha\left(\frac{q_{j}-q_{j-1}}{h_{j}}\right) f^{n-1}-\frac{A \alpha}{2}\left(f_{j}+f_{j-1}\right) q^{\prime n-1}=-\left[R_{1}\right]^{n-1} \\
& 4\left(1+\frac{R}{2}\right)\left(p_{j}-p_{j-1}\right)-4 R B \xi^{2} h_{j}\left(m_{j}+m_{j-1}\right)-\frac{\alpha}{2}\left(m_{j}+m_{j-1}\right) u^{n-1} \\
& -2 R B \xi^{2} h_{j}\left(v_{j}+v_{j-1}\right)+2 \xi h_{j}\left(p_{j}+p_{j-1}\right)+\frac{h_{j} \alpha}{2}\left(u_{j}+u_{j-1}\right) m^{n-1} \\
& +\frac{h_{j}(\alpha+7)}{4}\left(f_{j}+f_{j-1}\right)\left(p_{j}+p_{j-1}\right)-\frac{h_{j} \alpha}{2}\left(p_{j}+p_{j-1}\right) p^{n-1} \\
& -\frac{h_{j}(\alpha+1)}{4}\left(u_{j}+u_{j-1}\right)\left(m_{j}+m_{j-1}\right)=-\left[R_{2}\right]^{n-1} \\
& \frac{4}{\operatorname{Pr}}\left(t_{j}-t_{j-1}\right)+\frac{h_{j}(7+\alpha)}{4}\left(f_{j}+f_{j-1}\right)\left(t_{j}+t_{j-1}\right) \\
& +2 h_{j} \xi\left(t_{j}+t_{j-1}\right)-\frac{h_{j} \alpha}{4}\left(u_{j}+u_{j-1}\right)\left(s_{j}+s_{j-1}\right) \\
& +\frac{h_{j} \alpha}{2}\left(u_{j}+u_{j-1}\right) s^{n-1}-\frac{h_{j} \alpha}{2}\left(t_{j}+t_{j-1}\right) f^{n-1} \\
& +\frac{h_{j} \alpha}{2}\left(f_{j}+f_{j-1}\right) t^{n-1}-\frac{h_{j} \alpha}{2}\left(s_{j}+s_{j-1}\right) u^{n-1}=-\left[R_{3}\right]^{n-1} \\
& \text { Where, we have used the abbreviation } \alpha=\frac{\xi^{n-\frac{1}{2}}}{k_{n}}
\end{aligned}
$$

$$
\begin{aligned}
& \left(R_{1}\right)_{j-\frac{1}{2}}^{n-1}=h_{j}\left(\begin{array}{l}
\frac{4}{1+\lambda}+4(1+R) v^{\prime}-(\alpha-7) f v+4 R P \\
+(\alpha-2) u^{2}+4 s+4 \xi v-2 A(u q) \\
+A(\alpha-7) f q^{\prime}-4 A \xi q^{\prime}-A(\alpha-1) v^{2}
\end{array}\right) \\
& \left(R_{2}\right)_{j-\frac{1}{2}}^{n-1}=h_{j}\left(\begin{array}{l}
4\left(1+\frac{R}{2}\right) p^{\prime}-8 R B \xi^{2} m-4 R B \xi^{2} v \\
-(\alpha-7)(f p)+(\alpha-1)(u m)+4 \xi p
\end{array}\right) \\
& \left(R_{3}\right)_{j-\frac{1}{2}}^{n-1}=h_{j}\left[\frac{4}{\operatorname{Pr}} t^{\prime}-(\alpha-7) f t+\alpha(u s)+4 \xi t\right]
\end{aligned}
$$

The boundary conditions are

$f_{0}^{n}=u_{0}^{n}=0, s_{0}^{n}=1, m_{0}^{n}=1, u_{j}^{n}=0, v_{j}^{n}=0, s_{j}^{n}=0, m_{j}^{n}=0$

\section{Phase c: Quasi linearization of non-Linear Keller Algebraic Equations}

If we assume $f_{j}^{n-1}, u_{j}^{n-1}, v_{j}^{n-1}, s_{j}^{n-1}, t_{j}^{n-1}, q_{j}^{n-1}, m_{j}^{n-1}, p_{j}^{n-1}$ to be known for the solution of $8 \mathrm{~J}+8$ equations for the solution of $8 \mathrm{~J}+8$ unknowns $f_{j}^{n}, u_{j}^{n}, v_{j}^{n}, s_{j}^{n}, t_{j}^{n}, q_{j}^{n}, m_{j}^{n}, p_{j}^{n}, j=0,1,2, \ldots, J$. This non-linear system of algebraic equations is linearized by means of Newton's method as explained in (Keller 1970, Subba Rao et al. 2016)

\section{Phase d: Block-Tridiagonal Elimination of Linear Keller Algebraic Equations}

Linear system is solved using the block-elimination method, since it possess a block-tridiagonal The structure consists of variables or constants, but here an interesting feature can be observed, namely that it consists of block matrices the complete linearized system is formulated as a block matrix system, where each element in the co-efficient matrix is a matrix itself. Then, this system is solved using the efficient Kellerbox method. The numerical results are strongly influenced by the number of mesh points in both directions. After some trials in the $\eta$ directions (radial coordinate) a larger number of mesh points are selected, whereas in the $\xi$-directions (tangential coordinate) significantly less mesh points are necessary. The numerical algorithm is executed in MATLAB on a PC. The method demonstrates excellent stability, convergence and consistency, as elaborated by Keller (1970). coupled boundary layer equations in a $(\xi, \eta)$ coordinate system remain strongly nonlinear. A numerical method, the Keller-Box implicit difference method, is therefore deployed to solve the boundary value problem defined by Ens. (14) - (15) with boundary conditions (16). This technique has been described succinctly in Cebeci and Bradshaw (1984) and Keller (1970). It has been used recently in polymeric flow dynamics by Subba Rao et al. (2017) and Amanulla et al. (2017) for viscoelastic models. The key stages involved are as follows:

a. Reduction of the Nth order partial differential equation system to $\mathrm{N}$ first order equations

b. Finite difference discretization

c. Quasilinearization of non-linear Keller algebraic equations

d. Block-tridiagonal elimination of linear Keller algebraic equations

\section{NUMERICAL RESULTS AND DISCUSSION}

Comprehensive solutions have been obtained and are presented in Table 1-7 and Figs.3-10. The numerical problem comprises of two independent variables $(\xi, \eta)$, three dependent fluid dynamic variables $(f, \theta, \phi)$ and six thermo-physical and body force control parameter values are prescribed. 


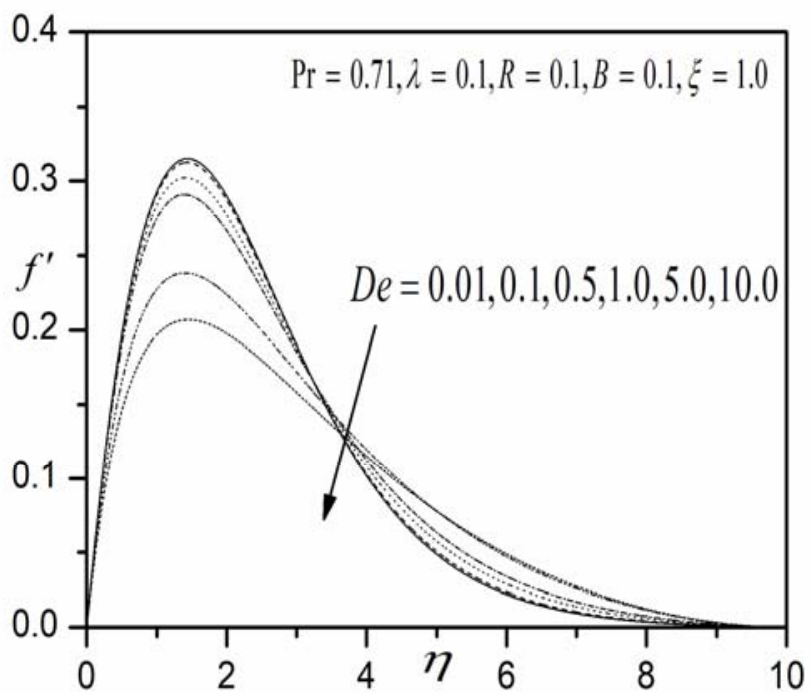

(a)

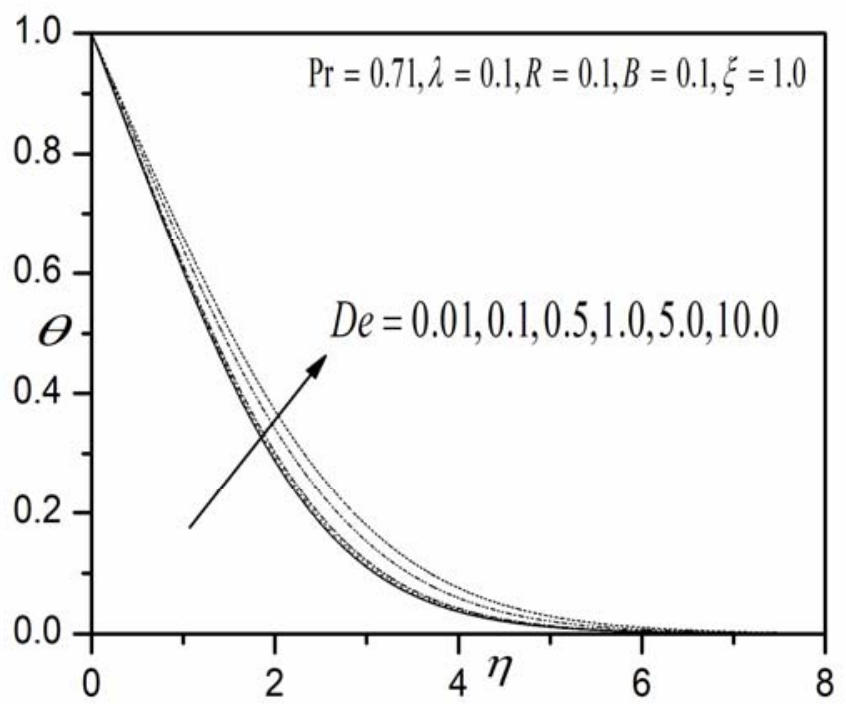

(b)

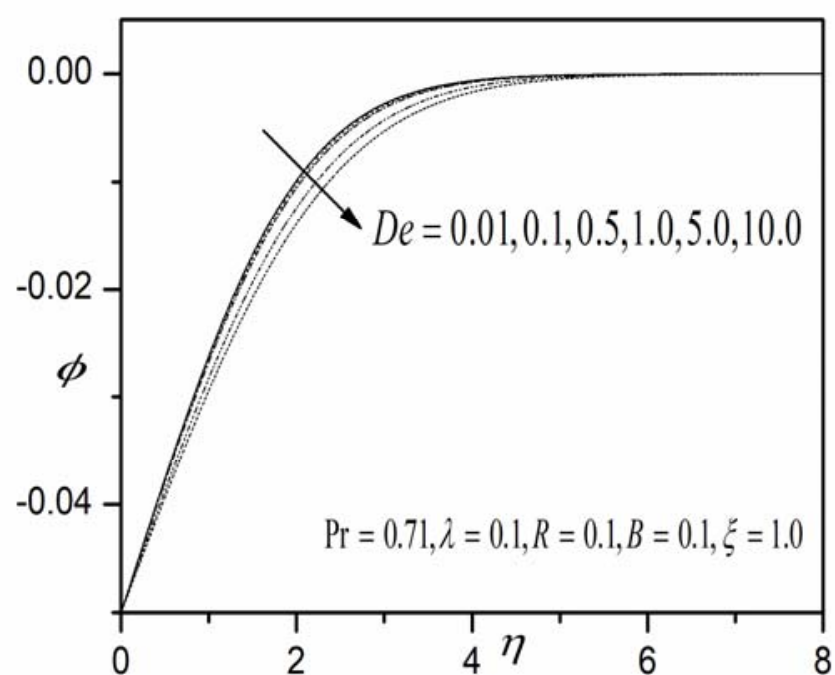

(c)

Fig. 3 Influence of De on (a) velocity profiles, (b) temperature profiles, and (c) angular velocity profiles.

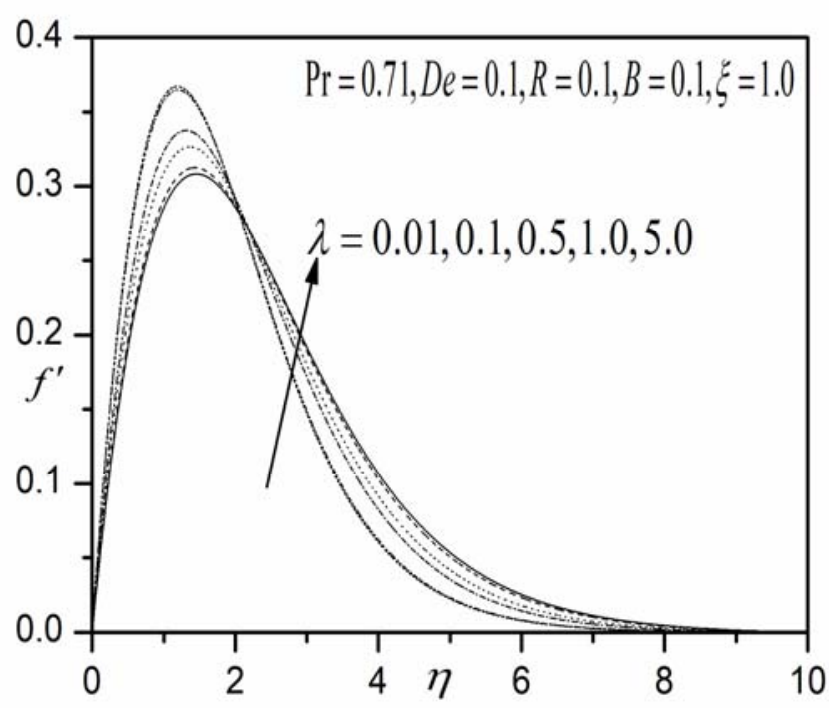

(a)

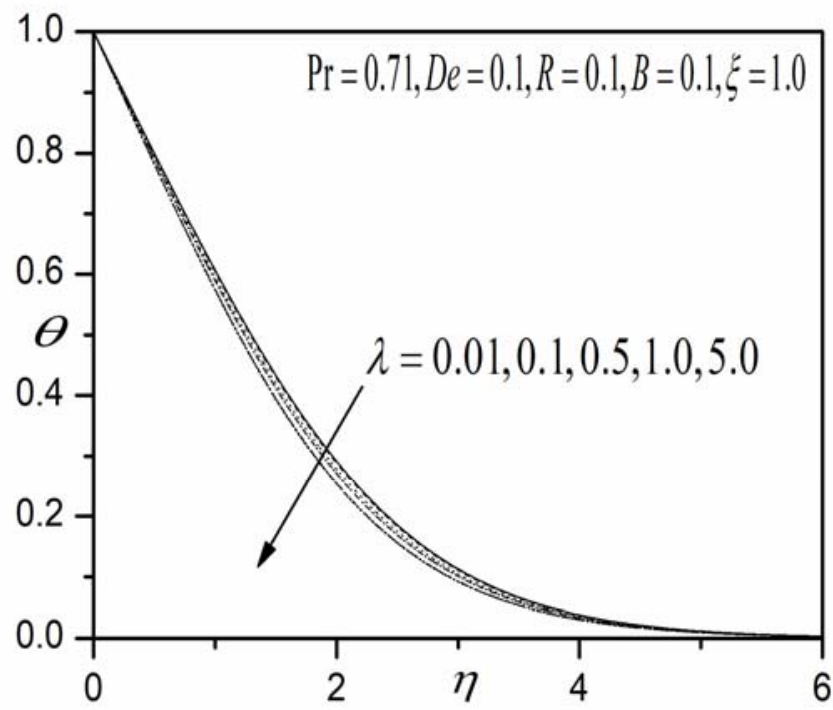

(b)

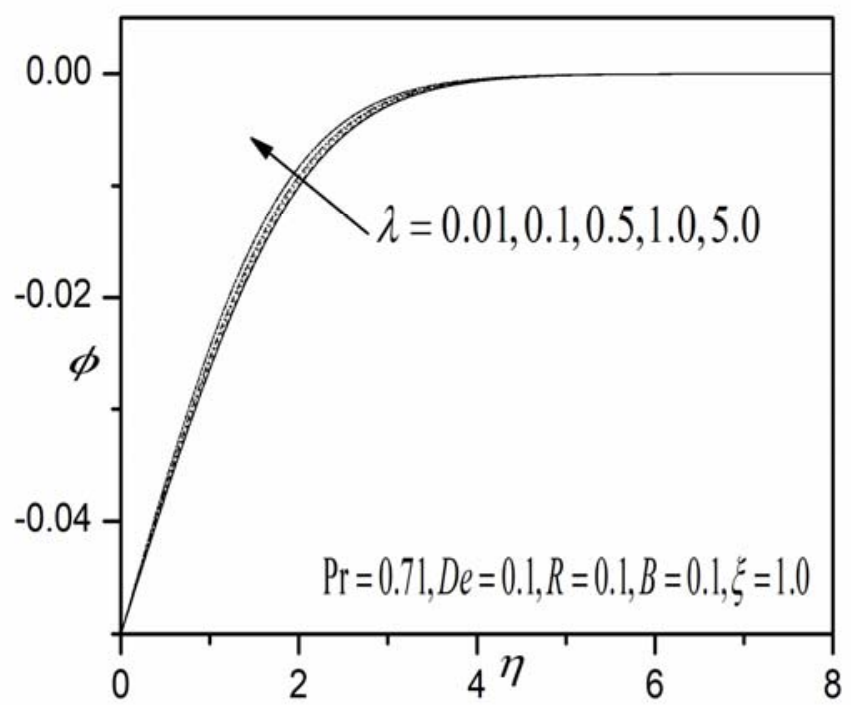

(c)

Fig. 4 Influence of $\lambda$ on the (a) velocity profiles, (b) temperature profiles, and (c) angular velocity profiles. 
Figs. 3(a) to 3(c), illustrate the influence of De on velocity $\left(f^{\prime}\right)$, temperature $(\theta)$ and angular velocity $(\phi)$. Dimensionless velocity component (fig.3) is considerably reduced with increasing De values. De arises in connection with some higher order derivatives in the momentum boundary layer equation (11),

i.e., $-\frac{D e}{1+\lambda}\left(2 f^{\prime \prime \prime \prime}-\left(f^{\prime \prime}\right)^{2}+7 f f^{i v}+4 \xi f^{i v}\right)$ and also

$\xi\left(-\frac{D e}{1+\lambda}\left(f^{\prime} \frac{\partial f^{\prime \prime \prime}}{\partial \xi}-f^{\prime \prime \prime} \frac{\partial f^{\prime}}{\partial \xi}+f^{\prime \prime} \frac{\partial f^{\prime \prime}}{\partial \xi}-f^{i v} \frac{\partial f}{\partial \xi}\right)\right)$. This parameter therefore exerts a strong influence on shearing characteristics of the polymer flow. The polymer behaves as a simple viscous fluid, as elaborated by Beg and Makinde (2011). For polymers, i.e., for non-Newtonian fluids, higher $D e$ values correspond to the polymer becoming highly oriented in one direction and stretched. For very high De value, the fluid movement is too fast for elastic forced to relax and the material acts as a purely elastic fluid. In fig. 3(c) with increasing De values, there is a substantial decrease in angular velocity.

Figs. 4(a) to 4(c) presents the effect of the ratio of relaxation to retardation times i.e., $\lambda$ on velocity $\left(f^{\prime}\right)$, temperature $(\theta)$ and angular velocity $(\phi)$ distributions throughout the boundary layer regime. A significant increase in velocity is observed with increasing $\lambda$ values. Conversely, temperature is markedly reduced with increasing values of $\lambda$. Also increasing $\lambda$ is seen to increase the angular velocity significantly. The mathematical model reduces to the Newtonian viscous flow model as $\lambda \rightarrow 0$ and $D e \rightarrow 0$.The momentum boundary layer equation in this cases contracts in the familiar Newtonian form $4(2+R) f^{\prime \prime \prime}+7 f f^{\prime \prime}+4 R g^{\prime}-2\left(f^{\prime}\right)^{2}+4 \theta+4 \xi f^{\prime \prime}=\xi\left(f^{\prime} \frac{\partial f^{\prime}}{\partial \xi}-f^{\prime \prime} \frac{\partial f}{\partial \xi}\right)$

Figs. 5(a) to 5(c) depicts the effect of the vortex viscosity parameter $R$ on velocity $\left(f^{\prime}\right)$, temperature $(\theta)$ and angular velocity $(\phi)$. For $R=1$, the micro-polar and Newtonian dynamic viscosity are equivalent

the micro-polar and Newtonian dynamic viscosity are equivalent. For $R=0$, micro-polarity is neglected and the equations reduce to Newtonian (Navier-Stokes) case. Although relatively simple in definition, $R$, has a prominent influence on all the flow variables. We observe in fig. 5(a) that an increase in $R$ strongly decelerates the flow, i.e., depresses linear velocity. Fig. 5(b) demonstrates that with stronger micro-polarity, i.e. greater $R$ value, the temperatures are elevated in the boundary layer. The regime is significantly heated and thermal boundary layer thickness is increased. The increased vortex viscosity encourages thermal diffusion and acts like as agitator. This increases the efficiency of thermal convection within the body of the fluid from the microscopic to the macroscopic scale and effectively transports heat with greater intensity of the cone surface into the fluid regime. Fig. 5(c) depicts that strong reversal of micro-element rotation is induced very close to the wall (cone surface) with increasing $R$ values.

Figs. 6(a) to 6(c) depicts the influence of velocity $\left(f^{\prime}\right)$, temperature $(\theta)$ and angular velocity $(\phi)$ for different values of material parameter B. It is observed that an increase of B significantly decelerates the flow, i.e., velocity throughout the boundary layer regime. The temperature also decreased with increasing $\mathrm{B}$ values. Whereas, an increasing the material parameter $\mathrm{B}$, the angular velocity is increased.

Figs. 7(a) to 7(c) represent typical profiles for velocity, temperature and angular velocity for various values of $\mathrm{Pr}$. It is observed that an increase the $\mathrm{Pr}$ leads to decrease in velocity. The most prominent variation in profiles arises at intermediate distances from the cone surface. Furthermore, increasing Pr generates a substantial reduction in the fluid temperature and the thermal boundary layer thickness. At large
Pr, the thermal boundary layer is thinner than at a smaller Pr. This is because for small values of $\operatorname{Pr}$, the fluid is highly conductive. Physically, if $\operatorname{Pr}$ increases, the thermal diffusivity decreases and these phenomena leads to the decreasing of energy, ability that reduces the thermal boundary layer. In addition, increasing Pr reduces angular velocity.

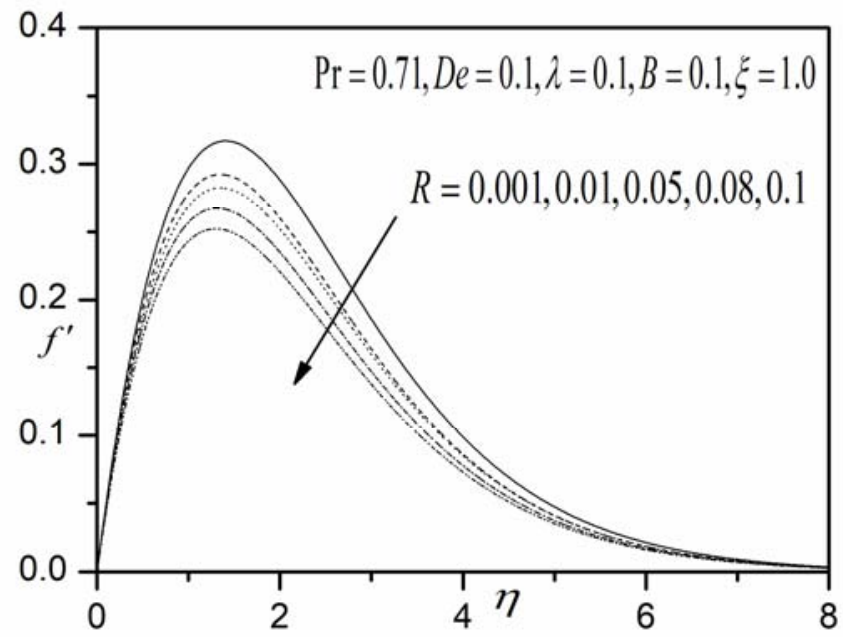

(a)

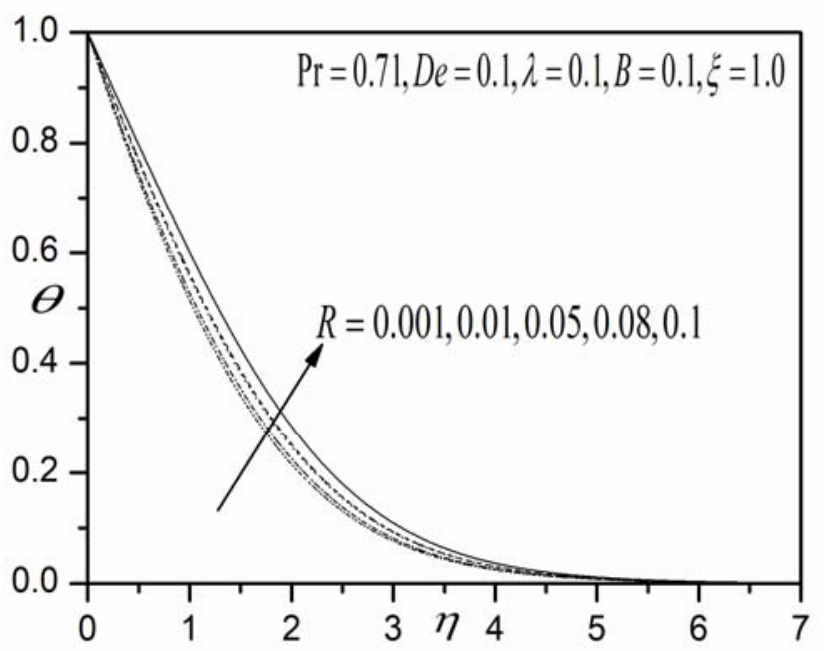

(b)

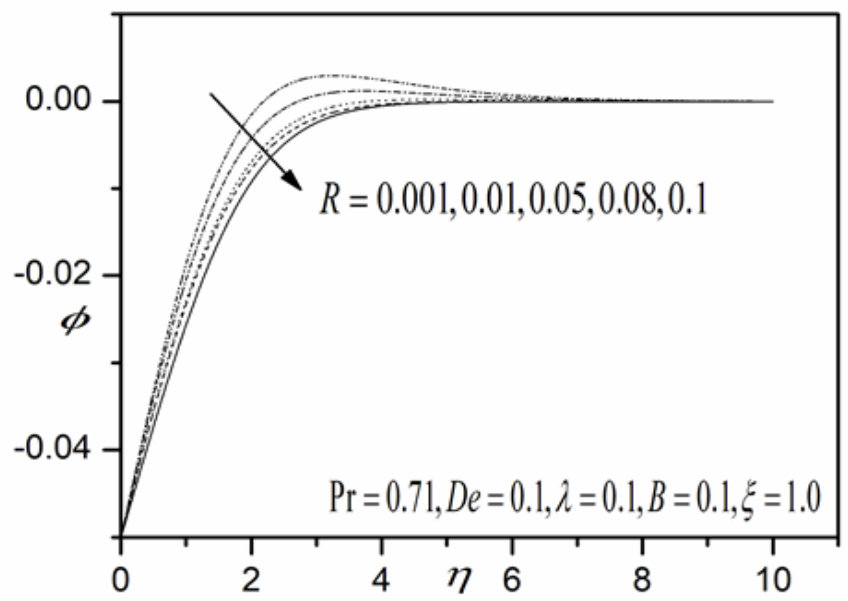

(c)

Fig. 5 Influence of R on the (a) velocity profiles, (b) temperature profiles, and (c) angular velocity profiles. 


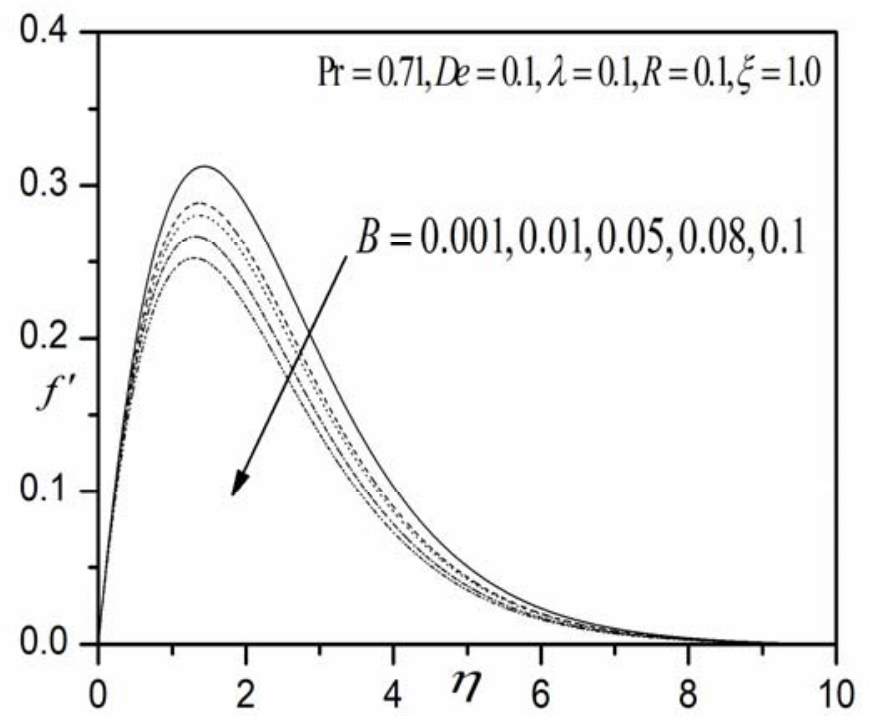

(a)

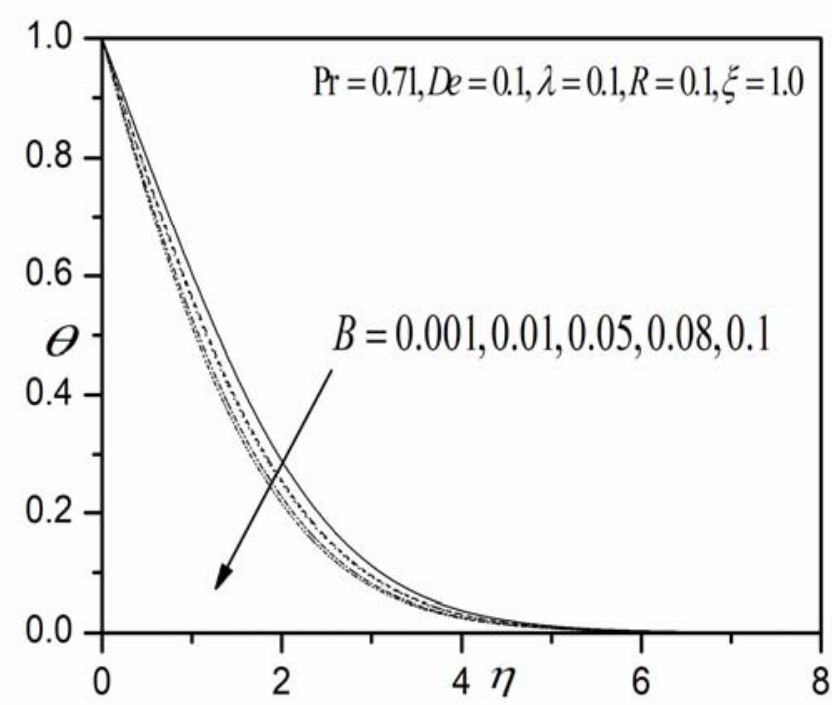

(b)

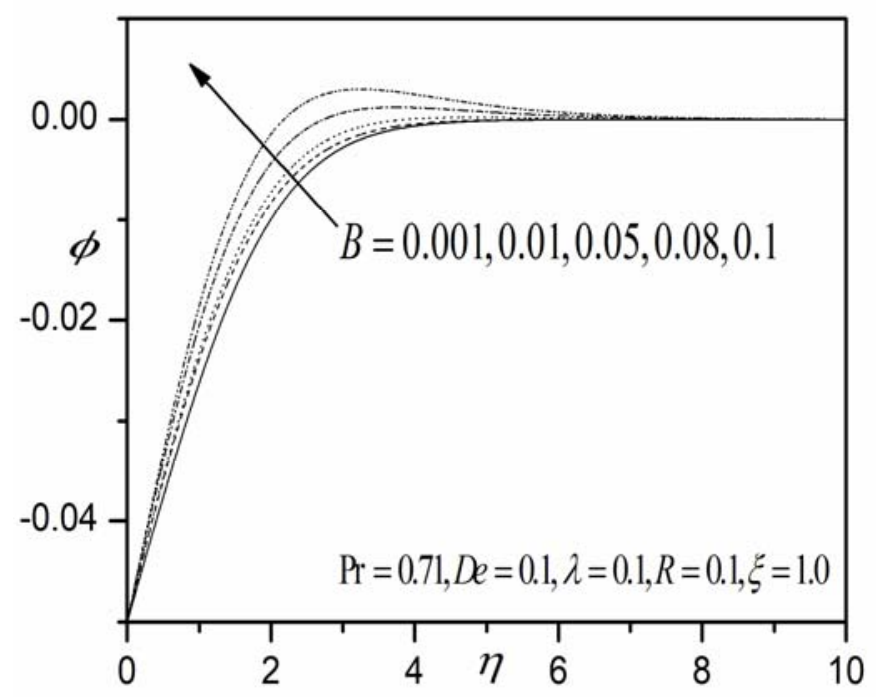

(c)

Fig. 6 Influence of B on the (a) velocity profiles, (b) temperature profiles, and (c) angular velocity profiles.

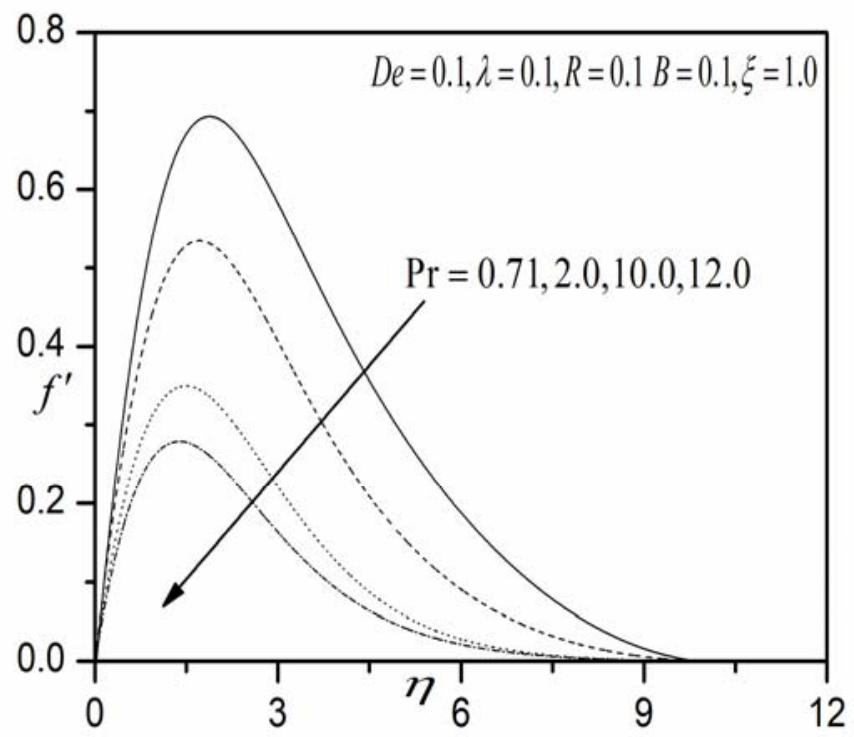

(a)

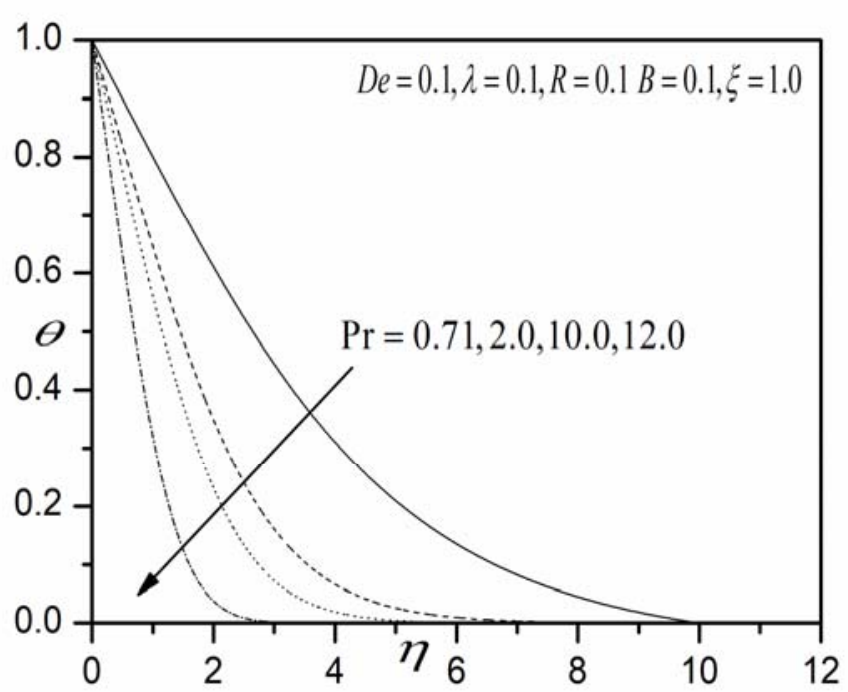

(b)

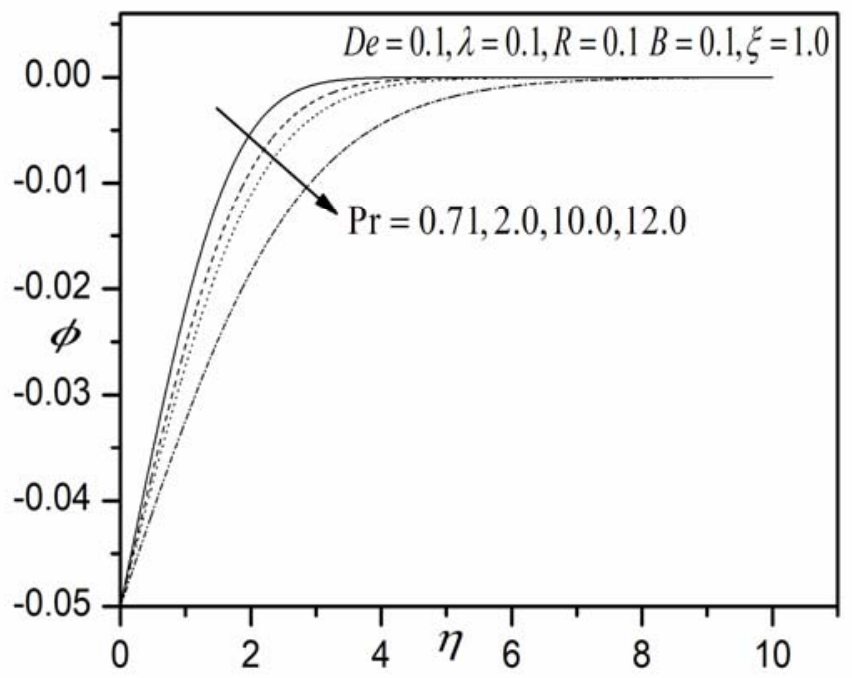

(c)

Fig. 7 Influence of Pr on the (a) velocity profiles, (b) temperature profiles, and (c) angular velocity profiles. 


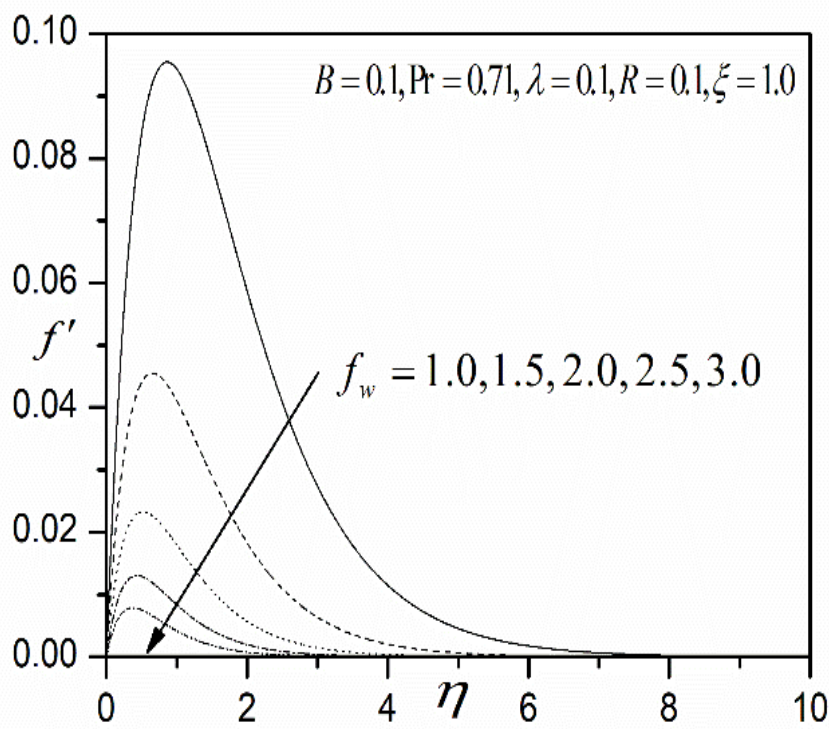

(a)

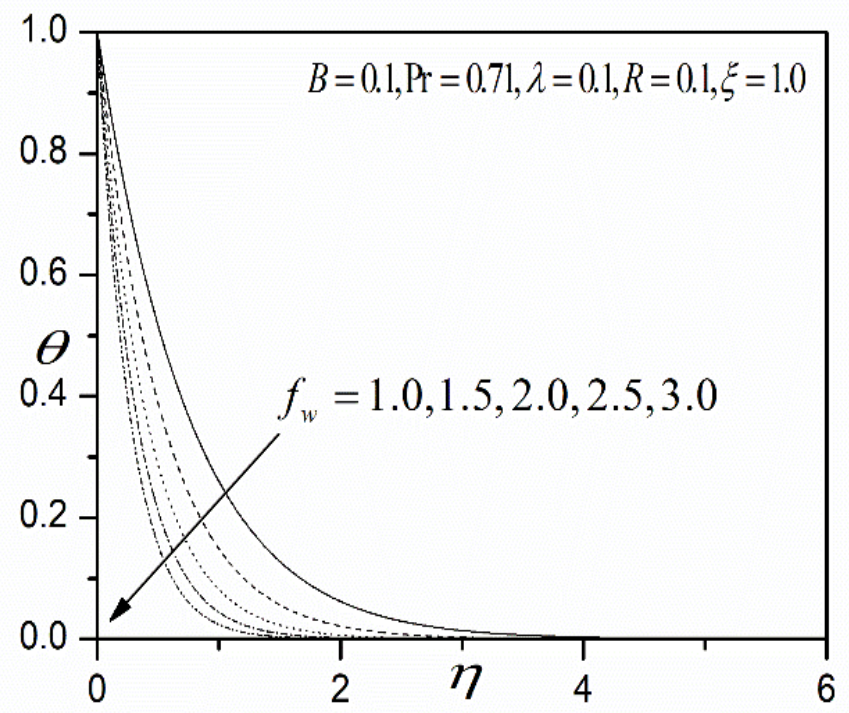

(b)

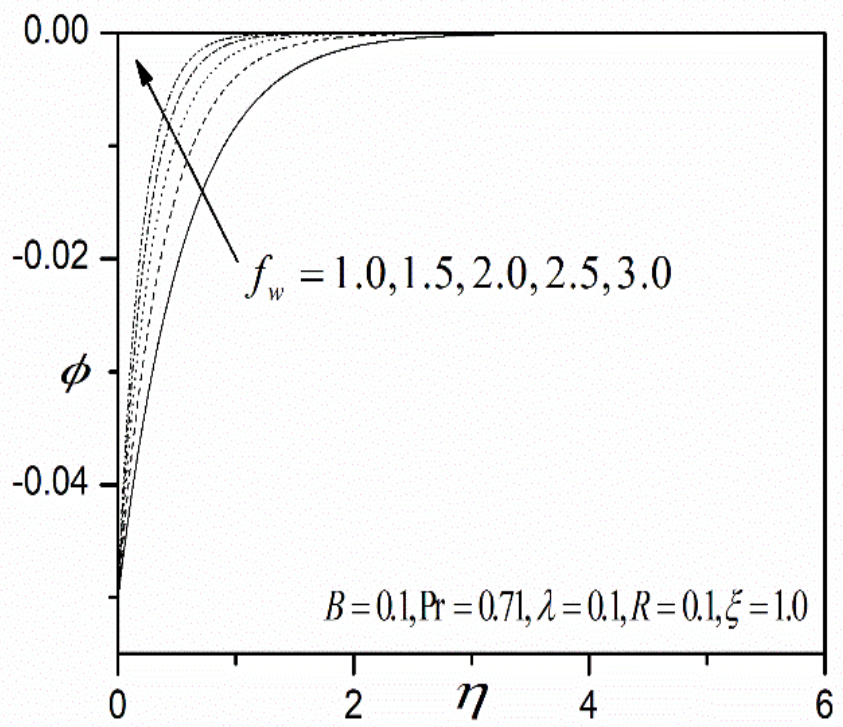

(c)

Fig. 8 Influence of $f_{w}$ on the (a) velocity profiles, (b) temperature profiles, and (c) angular velocity profiles.

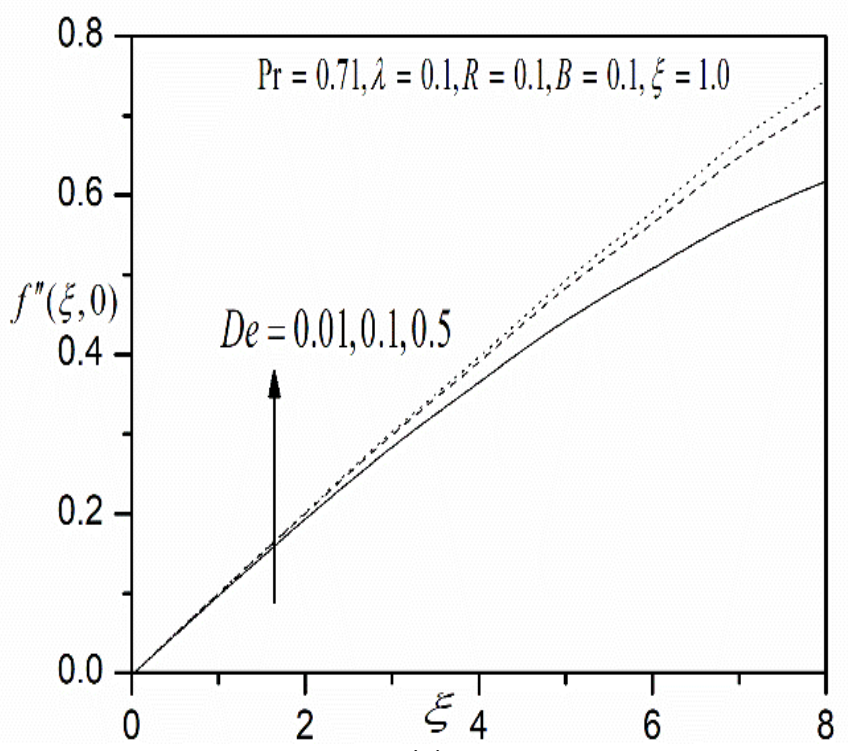

(a)

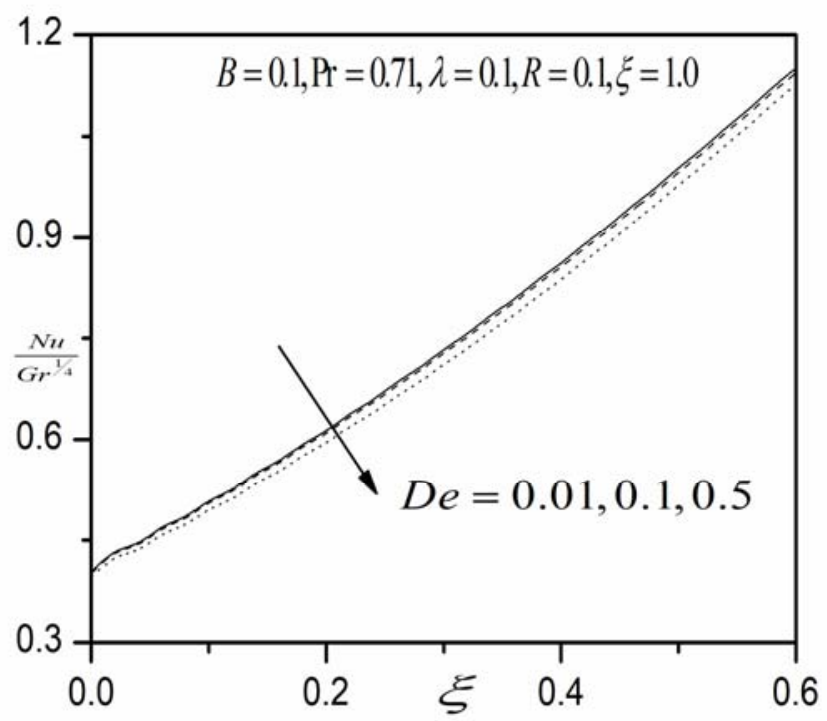

(b)

Fig.9 Influence of De on the (a) Skin friction coefficient and (b) Nusselt number coefficient.

Figs. 8(a) to 8(c) depicts the influence of velocity $\left(f^{\prime}\right)$, temperature $(\theta)$ and angular velocity $(\phi)$ for different values of suction parameter $\mathrm{f}_{\mathrm{w}}$. It is observed that an increase of $f_{w}$ significantly decelerates the flow, i.e., velocity throughout the boundary layer regime. The temperature also decreased with increasing $f_{w}$ values. Whereas, an increasing the suction parameter $f_{w}$, the angular velocity is increased.

Figs. 9 (a) to 9(b) presents the skin friction coefficient and Nusselt Number comparisons for different values of $D e$, Increasing Deborah number, $D e$ values first there was no increment after that skin friction increase and the decrease the Nusselt number throughout the cone surface..

Figs. 10 (a) to10 (b) presents the skin friction coefficient and Nusselt Number comparisons for different values of $R$, Increasing Vortex viscosity parameter, $R$ there was slow increment in the skin friction and also very slow decrement in the Nusselt number throughout the cone surface. 


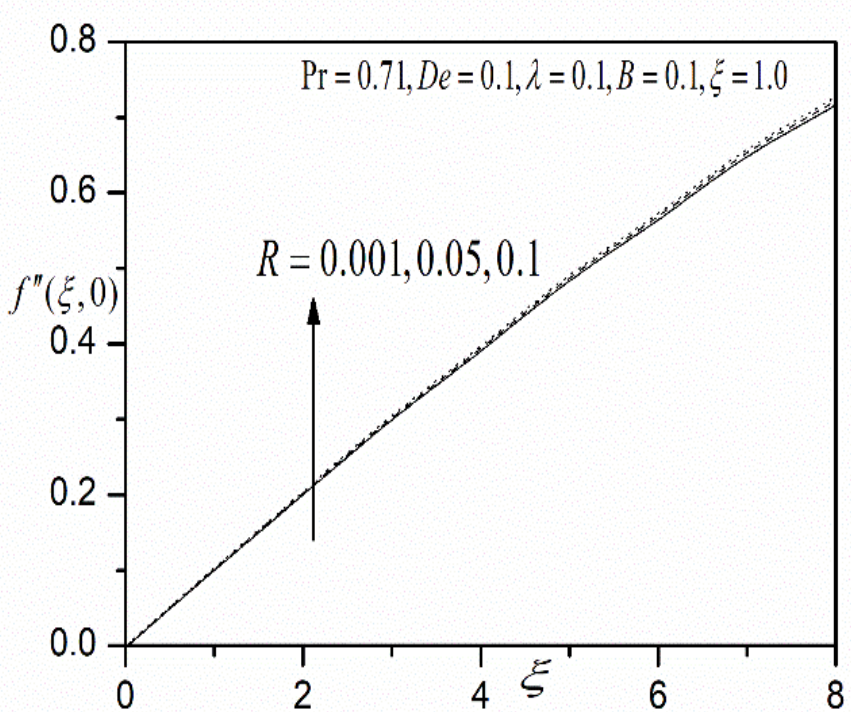

(a)

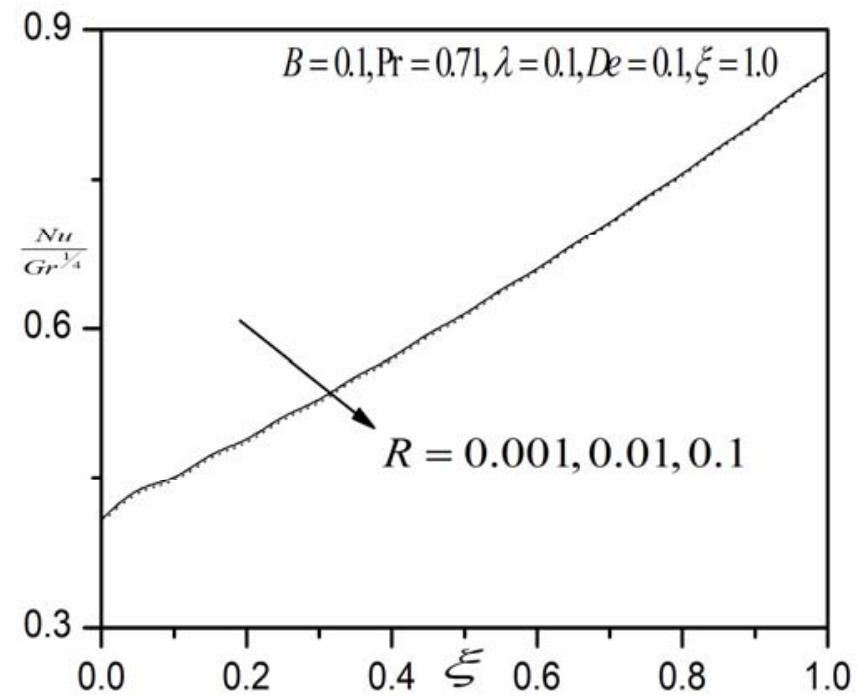

(b)

Fig. 10 Influence of $\mathrm{R}$ on the (a) Skin friction coefficient and (b) Nusselt number coefficient.

Table 1 Values of skin friction $\left(f^{\prime \prime}(\xi, 0)\right)$ for different $D e, \lambda$ and $\xi$

\begin{tabular}{|c|c|c|c|c|}
\hline \multirow{2}{*}{$D e$} & \multirow{2}{*}{$\lambda$} & \multicolumn{3}{|c|}{$f^{\prime \prime}(\xi, 0)$} \\
\hline & & $\xi=1.0$ & $\xi=2.0$ & $\xi=3.0$ \\
\hline 0.1 & \multirow{7}{*}{0.2} & 0.2515 & 0.4828 & 0.5419 \\
\hline 0.3 & & 0.2219 & 0.4353 & 0.4889 \\
\hline 0.5 & & 0.2022 & 0.4284 & 0.4487 \\
\hline 0.8 & & 0.1821 & 0.3573 & 0.401 \\
\hline 1.0 & & 0.1723 & 0.3357 & 0.376 \\
\hline 1.5 & & 0.1554 & 0.2954 & 0.3289 \\
\hline 2.0 & & 0.1456 & 0.268 & 0.2964 \\
\hline \multirow{6}{*}{0.1} & 0 & 0.2364 & 0.4488 & 0.503 \\
\hline & 0.5 & 0.2695 & 0.5235 & 0.5885 \\
\hline & 1.0 & 0.2915 & 0.5733 & 0.6454 \\
\hline & 2.0 & 0.3193 & 0.6359 & 0.717 \\
\hline & 3.0 & 0.3363 & 0.6741 & 0.7605 \\
\hline & 5.0 & 0.3562 & 0.7182 & 0.8107 \\
\hline
\end{tabular}

Table 2 Values of Nusselt number $\left(-\theta^{\prime}(\xi, 0)\right)$ for different $D e, \lambda$ and $\xi$

\begin{tabular}{|c|c|c|c|c|}
\hline \multirow{2}{*}{ De } & \multirow{2}{*}{$\lambda$} & \multicolumn{3}{|c|}{$-\theta^{\prime}(\xi, 0)$} \\
\hline & & $\xi=1.0$ & $\xi=2.0$ & $\xi=3.0$ \\
\hline 0.1 & \multirow{7}{*}{0.2} & 0.7684 & 0.8875 & 0.9229 \\
\hline 0.3 & & 0.7353 & 0.8507 & 0.9089 \\
\hline 0.5 & & 0.7303 & 0.8393 & 0.8979 \\
\hline 0.8 & & 0.7242 & 0.8255 & 0.8854 \\
\hline 1.0 & & 0.7205 & 0.8176 & 0.8789 \\
\hline 1.5 & & 0.7112 & 0.8006 & 0.8675 \\
\hline 2.0 & & 0.7009 & 0.7848 & 0.8616 \\
\hline \multirow{6}{*}{0.1} & 0 & 0.7683 & 0.882 & 0.9161 \\
\hline & 0.5 & 0.7686 & 0.8936 & 0.9304 \\
\hline & 1.0 & 0.7688 & 0.9006 & 0.9388 \\
\hline & 2.0 & 0.7691 & 0.9086 & 0.9484 \\
\hline & 3.0 & 0.7692 & 0.9132 & 0.9538 \\
\hline & 5.0 & 0.7694 & 0.9182 & 0.9597 \\
\hline
\end{tabular}

Table 3: Values of skin friction $\left(f^{\prime \prime}(\xi, 0)\right)$ for different $R$ and $B$

\begin{tabular}{|c|c|c|c|}
\hline \multirow{2}{*}{$R$} & \multicolumn{3}{|c|}{$f^{\prime \prime}(\xi, 0)$} \\
\cline { 2 - 4 } & $B=0.001$ & $B=0.01$ & $B=0.1$ \\
\hline 0.001 & 0.3516 & 0.2023 & 0.1723 \\
\hline 0.01 & 0.351 & 0.2018 & 0.172 \\
\hline 0.05 & 0.3488 & 0.2001 & 0.1706 \\
\hline 0.08 & 0.3462 & 0.198 & 0.1689 \\
\hline 0.1 & 0.3299 & 0.1847 & 0.1582 \\
\hline 0.2 & 0.316 & 0.1732 & 0.1489 \\
\hline 0.3 & 0.3058 & 0.1648 & 0.142 \\
\hline 0.4 & 0.2978 & 0.1582 & 0.1366 \\
\hline 0.5 & 0.2856 & 0.1482 & 0.1284 \\
\hline 1.0 & 0.1695 & 0.1349 & 0.1175 \\
\hline
\end{tabular}

Tables 3-4 presents the impact of vortex viscosity parameter $(\mathrm{R})$ on skin friction and Nusselt number coefficients along with a variation in material parameter $(B)$.With increasing $R$, it is detected that the heat transfer rate decreases significantly and skin friction is reduced which corresponds to a retardation of the boundary layer flow.

Tables 5-6 signifies the impression of Prandtl number (Pr) on skin friction and Nusselt number coefficients, along with a variation in ratio of relaxation to retardation times $(\lambda)$. With increasing $\operatorname{Pr}$, it is observed that the both skin friction and heat transfer rate increase significantly through the cone surface.

Table 7 illustrate the comparison of steady state Local Nusselt number for various values of $\xi$ with those of the work Hossain and Paul (2001), Ching-Yang Cheng (2008) and found to be in good agreement.

Table 4: Values of Nusselt number $\left(-\theta^{\prime}(\xi, 0)\right)$ for different $R$ and $B$

\begin{tabular}{|c|c|c|c|}
\hline \multirow{2}{*}{$R$} & \multicolumn{3}{|c|}{$-\theta^{\prime}(\xi, 0)$} \\
\cline { 2 - 4 } & $B=0.001$ & $B=0.01$ & $B=0.1$ \\
\hline 0.001 & 0.6685 & 0.6303 & 0.5205 \\
\hline 0.01 & 0.6683 & 0.6301 & 0.5204 \\
\hline 0.05 & 0.6675 & 0.6294 & 0.5198 \\
\hline 0.08 & 0.6667 & 0.6286 & 0.519 \\
\hline 0.1 & 0.6622 & 0.6239 & 0.5148 \\
\hline 0.2 & 0.6589 & 0.6205 & 0.5117 \\
\hline 0.3 & 0.6565 & 0.6186 & 0.5093 \\
\hline 0.4 & 0.6545 & 0.6174 & 0.5078 \\
\hline 0.5 & 0.6509 & 0.6162 & 0.5056 \\
\hline 1.0 & 0.645 & 0.6161 & 0.5036 \\
\hline
\end{tabular}


Table 5: Values of skin friction $\left(f^{\prime \prime}(\xi, 0)\right)$ for different $\operatorname{Pr}$ and $\lambda$

\begin{tabular}{|c|c|c|c|}
\hline \multirow{2}{*}{$\operatorname{Pr}$} & \multicolumn{3}{|c|}{$f^{\prime \prime}(\xi, 0)$} \\
\cline { 2 - 4 } & $\lambda=0.0$ & $\lambda=0.5$ & $\lambda=1.0$ \\
\hline 0.5 & 0.2364 & 0.2696 & 0.2916 \\
\hline 0.71 & 0.2359 & 0.269 & 0.2909 \\
\hline 5 & 0.2339 & 0.2665 & 0.2881 \\
\hline 7 & 0.2315 & 0.2636 & 0.2848 \\
\hline 10 & 0.2167 & 0.2456 & 0.2647 \\
\hline 15 & 0.2039 & 0.2304 & 0.2479 \\
\hline 25 & 0.1945 & 0.2193 & 0.2357 \\
\hline 50 & 0.1871 & 0.2106 & 0.2262 \\
\hline 75 & 0.1757 & 0.1974 & 0.2117 \\
\hline 100 & 0.1606 & 0.18 & 0.1928 \\
\hline
\end{tabular}

Table 6: Values of Nusselt number $\left(-\theta^{\prime}(\xi, 0)\right)$ for different $\operatorname{Pr}$ and $\lambda$

\begin{tabular}{|c|c|c|c|}
\hline \multirow{2}{*}{$\operatorname{Pr}$} & \multicolumn{3}{|c|}{$-\theta^{\prime}(\xi, 0)$} \\
\cline { 2 - 4 } & $\lambda=0.0$ & $\lambda=0.5$ & $\lambda=1.0$ \\
\hline 0.5 & 0.5483 & 0.6343 & 0.6398 \\
\hline 0.71 & 0.5981 & 0.6998 & 0.7018 \\
\hline 5 & 0.6675 & 0.7865 & 0.7865 \\
\hline 7 & 0.8667 & 0.8017 & 0.8450 \\
\hline 10 & 0.9123 & 0.9973 & 1.1597 \\
\hline 15 & 0.9893 & 1.1293 & 1.6701 \\
\hline 25 & 1.2371 & 1.5371 & 1.6743 \\
\hline 50 & 2.1152 & 2.4862 & 2.5670 \\
\hline 75 & 3.0019 & 3.5315 & 3.6219 \\
\hline 100 & 3.4466 & 3.7704 & 3.9863 \\
\hline
\end{tabular}

Table 7: Comparison table values of $N u$ for various values of $\xi$

\begin{tabular}{|c|c|c|c|}
\hline \multirow{2}{*}{$\xi$} & \multicolumn{3}{|c|}{$-\theta^{\prime}(\xi, 0)$} \\
\cline { 2 - 4 } & $\begin{array}{c}\text { Hossain } \\
\text { and Paul } \\
(2001)\end{array}$ & $\begin{array}{c}\text { Ching-Yang } \\
\text { Cheng } \\
(2008)\end{array}$ & Present \\
\hline 0 & 0.24584 & 0.2460 & 0.24642 \\
\hline 0.1 & 0.25089 & 0.2509 & 0.25091 \\
\hline 0.2 & 0.25601 & 0.2559 & 0.25563 \\
\hline 0.4 & 0.2663 & 0.2660 & 0.26591 \\
\hline 0.6 & 0.27662 & 0.2760 & 0.27661 \\
\hline 0.8 & 0.28694 & 0.2862 & 0.28696 \\
\hline 1 & 0.29731 & 0.2965 & 0.29734 \\
\hline 2 & 0.35131 & 0.3503 & 0.35137 \\
\hline
\end{tabular}

\section{CONCLUSIONS}

A mathematical model has been developed for the buoyancy-driven, non -similar, free convection boundary layer flow of Jeffery's nonNewtonian fluid from a vertical isothermal cone in a micro polar fluid regime. The transformed conservation equations have been solved with prescribed boundary conditions using the finite differences implicit Keller-box method. The present simulations have shown that

(i) Increase the Deborah number $(D e)$ decreases the velocity, angular velocity and Nusselt Number while in enhances the temperature and skin friction profiles.

(ii) Increase the vortex viscosity parameter $(R)$ in retards the velocity; temperature and the Nusselt number while enhance the skin friction and angular velocity profiles. (iii) Increase the Prandtl number (Pr) the velocity, temperature and angular velocity profiles are in decreases, while the Nusselt number increased.

(iv) Increase the suction parameter $\left(f_{w}\right)$ the velocity and temperature profiles are decreases throughout the cone surface and enhance the angular velocity profiles.

(v) Increase the material parameter $(B)$ the velocity and temperature profiles are decreases throughout the cone surface and enhance the angular velocity profiles.

\section{NOMENCLATURE}

A Half angle of the cone

$B \quad$ Micro polar inertia density (material) parameter

De Deborah number

$f \quad$ Dimensionless stream function

$g \quad$ Dimensionless stream function

$g^{*} \quad$ Gravitational acceleration

Gr Local Grashof number

$h \quad$ Heat transfer coefficient

j Micro inertia density

$k \quad$ Thermal conductivity

$N \quad$ Angular velocity

$\mathrm{Nu} \quad$ Nusselt number

Pr Prandtl number

$r \quad$ Local radius of the truncated cone

$R \quad$ Vortex viscosity parameter

$T \quad$ Temperature

$u, v \quad$ Dimensionless velocity components along $\mathrm{x}$ and $\mathrm{y}$ directions respectively

$\mathrm{x} \quad$ stream wise coordinate

y Transverse coordinate

\section{Greek Symbols}

$\begin{array}{ll}\alpha & \text { Thermal diffusivity } \\ \beta & \text { Coefficient concentration expansion } \\ \gamma^{*} & \text { Spin gradient viscosity } \\ \xi, \eta & \text { Dimensionless coordinates } \\ \theta & \text { Dimensionless temperature } \\ \lambda & \text { Ratio of retardation time to relaxation time } \\ \lambda_{1} & \text { retardation time } \\ k & \text { Vortex viscosity } \\ \mu & \text { Viscosity } \\ \rho & \text { Density } \\ \psi & \text { Dimensionless stream function }\end{array}$

\section{Subscripts}

w conditions on the wall

$\infty \quad$ free stream conditions

\section{ACKNOWLEDGEMENTS}

Authors thanks to DST [Ref: N0. SR/WOS-A/MS-09/2014(G)] New Delhi, for financial support and management of Madanapalle Institute of Technology \& science, Madanapalle for providing research facilities in the campus. 


\section{REFERENCES}

Abbasi, F.M., Shehzad, A., Hayat, T., and Alhumali, M.S., 2016, "Mixed Convection Flow of Jeffrey Nano Fluid with Thermal Radiation and Double Stratification," Journal of Hydrodynamics, 28(5) 840-849. https://doi.org/10.1016/S1001-6058(16)60686-8

Abid, H., Mohd, Z.S., Ilyas, K., and Shafie, S., 2017, "Convection Heat Transfer in Micropolar Nano Fluids with Oxide Nano Particles in Water, Kerosene and Engine Oil," J. Mol. Liq., 229, 482-488. https://doi.org/10.1016/i.molliq.2016.12.040

Amanulla, C.H., Nagendra, N., Surya Narayana Reddy, M., Subba Rao, A., and Anwar Bég, O., 2017, "Mathematical Study of Non-Newtonian Nanofluid Transport Phenomena from an Isothermal Sphere," Frontiers in Heat and Mass Transfer, 8, 29.

http://dx.doi.org/10.5098/hmt.8.29

Amanulla, C.H., Nagendra, N., Subba Rao A., Anwar Bég O., and Kadir A., 2017, "Numerical Exploration of Thermal Radiation and Biot Number Effects on the Flow of a Non-Newtonian MHD Williamson Fluid over a Vertical Convective Surface," Heat Trans Asian Res. 00, $1-19$.

https://doi.org/10.1002/htj.21303

Amanulla, C.H., Nagendra, N., and Suryanarayana Reddy, M., 2017, "MHD Flow and Heat Transfer in a Williamson Fluid from a Vertical Permeable Cone with Thermal and Momentum Slip Effects: A Mathematical Study," Frontiers in Heat and Mass Transfer, 8, 40. http://dx.doi.org/10.5098/hmt.8.40

Amanulla, C.H., Nagendra, N., and Suryanarayana Reddy, M., 2017, "Multiple Slip Effects on MHD and Heat Transfer in a Jeffery Fluid over an Inclined Vertical Plate," International Journal of Pure and Applied Mathematics, 113(7), 137-145.

Amanulla, C.H., Nagendra, N., and Surya Narayana Reddy, M., 2017, "Numerical Study of Thermal and Momentum Slip Effects on MHD Williamson Nanofluid from an Isothermal Sphere," Journal of Nanofluids, 6(6), 1111-1126.

https://doi.org/10.1166/jon.2017.1405

Amanulla, CH., Nagendra, N., Surya Narayana Reddy, M., Subba Rao, A., and Anwar Bég, O., 2017, "Thermal and Momentum Slip Effects on Hydromagnetic Convection Flow of a Williamson Fluid Past a Vertical Truncated Cone," Frontiers in Heat and Mass Transfer, 9, 22. https://doi.org/10.5809/hmt.9.22

Amanulla, C.H., Nagendra, N., and Suryanarayana Reddy, M., 2017, "Computational Analysis of Non-Newtonian Boundary Layer Flow of Nanofluid Past a Semi-infinite Vertical Plate with Partial Slip," Nonlinear Engineering, 1-15.

https://doi.org/10.1515/nleng-2017-0055

Aparna, P., Pothanna, N., Murthy, J.V.R., and. Sreelatha, K., 2017, "Flow Generated by Slow Steady Rotation of a Permeable Sphere in a Micropolar Fluid", Original Research Article, Alexandria Eng. J. https://doi.org/10.1016/j.aej.2017.01.018

Ashmawy, E.A., 2015, "Fully Developed Natural Convective Micropolar Fluid Flow in a Vertical Channel with Slip", J. Egyptian Mathematical Society, 23, 563-567.

https://doi.org/10.1016/j.joems.2014.06.019

Bég, O.A., Prasad, V.R., Vasu, B., and Bhaskar Reddy, N., 2011, "Free Convection Heat and Mass Transfer from an Isothermal Sphere to a Micropolar Regime with Soret/Dufour Effects," Int. J. Heat and MassTransfer.,54, 9-18. https://doi.org/10.1016/i.iiheatmasstransfer.2010.10.005

Bég, O.A, and Makinde. O.D., 2011, "Viscoelastic Flow and Species Transfer in a Darcian High-Permeability Channel," J. Petroleum Sci. and Eng., 76, 93-99.

https://doi.org/10.1016/j.petrol.2011.01.008

Asia, Y., Kashif, A., and Muhammad, A., 2016, "MHD Unsteady Flow and Heat Transfer of Micropolar Fluid through Porous Channel with Expanding or Contracting Walls," J. Applied Fluid Mechanics, 9, 18071817.

http://doi.org/10.18869/acadpub.jafm.68.235.22857

Aurangzaib, Uddin, Md.S. Bhattacharyya, K., and Shafie, S., 2016, "Micropolar Fluid Flow and Heat Transfer over an Exponentially Permeable Shrinking Sheet," Propulsion and Power Research, 5(4), 310-317.

https://doi.org/10.1016/j.jppr.2016.11.005

Aurangzaib, Bhattacharyya, K., and Shafie, S., 2016, "Effect of Partial Slip on an Unsteady MHD Mixed Convection Stagnation-Point Flow of a Micropolar Fluid towards a Permeable Shrinking Sheet," Alexandria Engineering Journal, 55(2), 1285-1293.

https://doi.org/10.1016/j.aej.2016.04.018

Baag, S., Mishra, S.R., Dash, G.C., and Acharya, M., 2017, "Numerical Investigation on MHD Micropolar Fluid Flow toward a Stagnation Point on a Vertical Surface with Heat Source and Chemical Reaction," J. King Saud University Eng. Sci., 29, 75-83. https://doi.org/10.1016/j.jksues.2014.06.002

Bourantas, G.C., and Loukopoulos, V.C., 2014 "MHD NaturalConvection Flow in an Inclined Square Enclosure Filled with a Micropolar-Nano Fluid," Int. J. Heat and Mass Transfer, 79, 930-944. https://doi.org/10.1016/j.ijheatmasstransfer.2014.08.0755

Cheng-Long Chang., 2008, "Numerical Simulation for Natural Convection of Micropolar Fluids Flow along Slender Hollow Circular Cylinder with Wall Conduction Effect," Comm. in Nonlinear Sci. and Numerical Simulation., 13,624-636.

https://doi.org/10.1016/j.cnsns.2006.05.012

Ching-Yang Cheng, 2006, "Free Convection Heat and Mass Transfer from a Horizontal Cylinder of Elliptic Cross Section in Micropolar Fluids", Int. Commu. in Heat and MassTransfer, 33, 313-318. https://doi.org/10.1016/j.icheatmasstransfer.2005.10.012

Ching- Yang Cheng. ,2008, "Natural Convection Heat and Mass Transfer from a Sphere in Micropolar Fluids with Constant Wall Temperature and Concentration," Int. Comm. Heat and Mass Trans., 35, 750-755.

https://doi.org/10.1016/j.icheatmasstransfer.2008.02.004

Ching-Yang Cheng., 2008, "Natural Convection of a Micropolar Fluid from a Vertical Truncated Cone with Power-law Variation in Surface Temperature," Int. Comm. Heat and Mass Trans., 35, 39- 46. https://doi.org/10.1016/j.icheatmasstransfer.2007.05.018

Dulal Pala, Gopinath Mandalb., 2017, "Thermal Radiation and MHD Effects on Boundary Layer Flow of Micropolar Nanofluid Past a Stretching Sheet with Non-Uniform Heat Source/Sink," Int. J. Mech. Sci., 126, 308-318.

https://doi.org/10.1016/j.ijmecsci.2016.12.023

Hari, R., Kataria., Harshad.R., Patel, Rajiv Singh, 2017, "Effect of Magnetic Field on Unsteady Natural Convective Flow of a Micropolar 
Fluid Between Two Vertical Walls," Ain Shams Eng. J., 8, 87-102. https://doi.org/10.1016/j.asej.2015.08.013

Hayat, T., Sadia A., Mustafac, M., and Alsaedib, A., 2014. "MHD Stagnation-Point Flow of Jeffrey Fluid over a Convectively Heated Stretching Sheet," Computers \& Fluids, 108, 179-185.

https://doi.org/10.1016/j.compfluid.2014.11.016

Hossain, M.A., and Paul, S.C., 2001, "Free Convection from a Vertical Permeable Circular Cone with Non-Uniform Surface Temperature," Acta Mechanica, 151,103- 114.

Isaac Lare Animasaun., 2017, “ Melting Heat and Mass Transfer in Stagnation Point Micropolar Fluid Flow of Temperature Dependent Fluid Viscosity and Thermal Conductivity at Constant Vortex Viscosity," J. Egyptian Mathematical Soc., 25, 79-85.

http://dx.doi.org/10.1016/j.joems.2016.06.007

Keller, H.B., 1970. "A New Difference Method for Parabolic Problems," J. Bramble (Editor), Numerical Methods for Partial Differential Equations, Academic Press, New York, USA

Mishra, S.R., Pattnaikand, P.K., and Dash, G.C., 2015, "Effect of Heat Source and Double Stratification on MHD Free Convection in a Micropolar Fluid," Alexandria Eng. J., 4, 681-689.

http://dx.doi.org/10.1016/j.aej.2015.04.010

Molla, M.M., Paul, S.C., and Hossain, M.A.,2009, "Natural Convection Flow from a Horizontal Circular with Uniform Heat flux in Presence of Heat Generation", Appl. Math. Model. 33, 3226-3236. http://dx.doi.org/10.1016/j.apm.2008.10.039

Nagendra, N., Subba Reddy, M.V., and Jayaraj, B., 2008, "Peristaltic Motion of a Power-law Fluid in an Asymmetric Vertical Channel," Journal of Interdisciplinary Mathematics, 11, 4, 505-519.

http://dx.doi.org/10.1080/09720502.2008.10700577

Nasir Ali, Sami Ullah Khan, Muhammad Sajid and Zaheer Abbas, 2016, "MHD Flow and Heat Transfer of Couple Stress Fluid Over an Oscillatory Stretching Sheet with Heat Source/Sink in Porous Medium," Alexandria Eng. J., 55, 915-924.

https://doi.org/10.1016/j.aej.2016.02.018

Naveed, M., Abbas, Z., and Sajid, M., 2016, "MHD Flow of Micropolar Fluid due to a Curved Stretching Sheet with Thermal Radiation," $J$. Applied. Fluid Mechanics, 9, 131-138. https://doi.org/10.1016/j.molliq.2016.01.012

Rashad, A.M., Abbasbandy, S., and Chamkha, A.J., 2014, "Mixed Convection Flow of a Micropolar Fluid over a Continuously Moving
Vertical Surface Immersed in a Thermally and Solutally Stratifyed Medium with Chemical Reaction," J. Taiwan Institute of Chemical Engineers, 45, 2163-2169.

http://dx.doi.org/10.1016/j.jtice.2014.07.002

Rawat. S., Kapoor, S., and Bhargava, R., 2016, “ MHD Flow Heat and Mass Transfer of Micropolar Fluid over a Nonlinear Stretching Sheet with Variable Micro Inertia Density Heat Flux and Chemical Reaction in a Non-Darcy Porous Medium," J. Applied Fluid Mechanic, 9, 321331 .

Siddiqa,S., Faryad, A., Naheed Begum, A.,.Hossain, M.A., Hossain and Rama Subba Gorla., 2017, "Periodic Magneto Hydrodynamic Natural Convection Flow of a Micropolar Fluid with Radiation," Int. J. Thermal Sciences, 111, 215-222.

https://doi.org/10.1016/j.ijthermalsci.2016.09.002

Satya Narayana, P.V., and Venkateswarlu, B., 2015, "Effects of Thermal Radiation on Unsteady MHD Micropolar Fluid Past a Vertical Porous Plate in the Presence of Radiation Absorption," International Journal of Engineering Science and Computing, 6, 9, 2259-2270.

Siddiqa, M.K.., Raufb, A., Shehzadb, S.A., Abbasic, F.M., and Merajb, M.A., 2017, “ Thermally and Solutally Convective Radiation in MHD Stagnation Point Flow of Micropolar Nanofluid over a Shrinking Sheet," Alexandria Eng. J., 4. (In Press) https://doi.org/10.1016/j.aej.2017.01.019

Srinivas Acharya, D., and Upendar, M., 2013, "Effect of Double Stratification on MHD Free Convection in a Micropolar Fluid," $J$. Egyptian Mathematical Soc., 21, 370-378.

http://dx.doi.org/10.1016/j.joems.2013.02.006

Subba Rao, A., Nagendra, N., and Prasad, V.R., 2015, "Heat Transfer in a Non-Newtonian Jeffrey's Fluid over a Non-Isothermal Wedge," Procedia Engineering, 127, 775-782.

https://doi.org/10.1016/j.proeng.2015.11.412

Subba Rao, A., Prasad, V.R., Nagendra, N., Bhaskar Reddy, N., and Anwar Beg, O., 2016, "Non-Similar Computational Solution for Boundarylayer Flows of Non-Newtonian Fluid from an Inclined plate with Thermal Slip," Journal of Applied Fluid Mechanics, 9(2), 795807.

Vanita and Anand Kumar., 2016, "Numerical Study of Effect of Induced Magnetic Field on Transient Natural Convection over a Vertical Cone", Alexandria Engineering Journal, 55, 1211-1223. https://doi.org/10.1016/j.aej.2016.04.007 